\title{
Profinite completions of orientable Poincaré duality groups of dimension four and Euler characteristic zero
}

\author{
Dessislava H. Kochloukova*
}

\begin{abstract}
Let $p$ be a prime number, $\mathcal{T}$ a class of finite groups closed under extensions, subgroups and quotients, and suppose that the cyclic group of order $p$ is in $\mathcal{T}$.

We find some sufficient and necessary conditions for the pro- $\mathcal{T}$ completion of an abstract orientable Poincaré duality group $G$ of dimension 4 and Euler characteristic 0 to be a profinite orientable Poincaré duality group of dimension 4 at the prime $p$ with Euler $p$-characteristic 0 . In particular we show that the pro- $p$ completion $\widehat{G}_{p}$ of $G$ is an orientable Poincaré duality pro- $p$ group of dimension 4 and Euler characteristic 0 if and only if $G$ is $p$-good.
\end{abstract}

Mathematics Subject Classification (2000). 20E18, 20J05.

Keywords. Poincaré duality group, profinite completion, $p$-good group.

\section{Introduction}

In this paper we study pro- $\mathcal{T}$ completions of abstract Poincaré duality groups of dimension 4 with Euler characteristic 0, where $\mathcal{T}$ is a class of finite groups that is subgroup, extension and quotient closed and the cyclic group of order $p$ is in $\mathcal{T}$ for a fixed prime $p$. This paper can be considered as a natural continuation of an earlier paper where profinite and pro- $p$ completions of an abstract orientable Poincaré duality group $G$ of dimension 3 were studied [6].

One of the results obtained in [6] is an algebraic proof of the Reznikov's claim that the pro- $p$ completion of the fundamental group of a closed orientable hyperbolic 3-manifold that violates the Thurston Conjecture is an orientable pro- $p$ Poincare duality group provided the pro- $p$ completion is infinite [11]. A quite different proof of the same claim was independently discovered by T. Weigel [16].

We call a profinite group a strong $\mathrm{PD}_{n}$ group at $p$ if it is a profinite Poincaré duality group of dimension $n$ at $p$ according to the definition of [15] and keep the name of profinite $\mathrm{PD}_{n}$ group at $p$ for groups satisfying the original Tate's definition [10], [14]. We discuss in details both definitions in the preliminaries.

\footnotetext{
*Partially supported by "bolsa de produtividade de pesquisa" from CNPq, Brazil.
} 
In the case of pro- $p$ groups both definitions are equivalent, but it is not known whether they are equivalent in general.

Theorem 1. Let $p$ be a prime number and $G$ be an abstract orientable Poincaré duality group of dimension 4 and Euler characteristic $\chi(G)=0$.

Let $\mathcal{T}$ be a class of finite groups closed under subgroups, extensions and quotients, let the cyclic group of order $p$ be in $\mathcal{T}$ and let $\mathcal{C}$ be a directed set of normal subgroups of finite index in $G$ such that $\mathcal{C}$ induces the pro- $\mathcal{T}$ topology of $G$.

Then

$$
\widehat{G}_{e}=\lim _{U \in \mathcal{C}} G / U
$$

is an orientable profinite Poincaré duality group of dimension 4 at the prime $p$ with Euler $p$-characteristic $\chi_{p}\left(\widehat{G}_{e}\right)=0$ if and only if all of the following conditions hold:

a) $\operatorname{cd}_{p}\left(\hat{G}_{e}\right)$ is finite and the Sylow p-subgroups of $\widehat{G}_{e}$ are not free or trivial, i.e., $2 \leq \operatorname{cd}_{p}\left(\widehat{G}_{\ell}\right)<\infty$

b) for every $U \in \mathcal{C}$ we have $\sum_{0 \leq i \leq 4}(-1)^{i} \operatorname{dim}_{\mathbb{F}_{p}} H_{i}\left(\hat{U}_{\mathcal{C}}, \mathbb{F}_{p}\right)=0$;

c) for every $U \in \mathcal{C}$ we have $2 \operatorname{dim}_{\mathbb{F}_{p}} H_{1}\left(\hat{U}_{e}, \mathbb{F}_{p}\right)-\operatorname{dim}_{\mathbb{F}_{p}} H_{2}\left(\hat{U}_{\mathcal{C}}, \mathbb{F}_{p}\right)=2$.

Furthermore, if the conditions a), b) and c) hold, then $\hat{G}_{e}$ is a strong profinite orientable Poincaré duality group of dimension 4 at $p$.

Remarks. 1. Since $\infty>\operatorname{cd}_{p}\left(\widehat{G}_{\ell}\right) \geq 1$ every Sylow $p$-subgroup of $\widehat{G}_{\ell}$ is infinite.

2. If condition a) is substituted with $2 \leq \operatorname{cd}_{p}\left(\hat{G}_{e}\right) \leq 4$ condition b) can be substituted with $\chi_{p}\left(\hat{G}_{\complement}\right)=0$, since $0=\chi_{p}\left(\hat{U}_{\complement}\right)=\sum_{0 \leq i \leq 4}(-1)^{i} \operatorname{dim}_{\mathbb{F}_{p}} H_{i}\left(\hat{U}_{\complement}, \mathbb{F}_{p}\right)$.

3. Theorem 1 implies that if conditions a), b) and c) hold then the only possibility for $\operatorname{cd}_{p}\left(\widehat{G}_{p}\right)$ is 4 .

An abstract group $G$ is said to be good if the natural map between continuous and abstract cohomology $H^{i}(\widehat{G}, M) \rightarrow H^{i}(G, M)$ is an isomorphism for every finite discrete $G$-module $M$, where $\widehat{G}$ is the profinite completion of $G$. The group $G$ is $p$-good if $H^{i}\left(\widehat{G}_{p}, M\right) \rightarrow H^{i}(G, M)$ is an isomorphism for every $p$-primary finite discrete $\widehat{G}_{p}$ - module $M$, where $\widehat{G}_{p}$ is the pro- $p$ completion of $G$.

Theorem 1 easily implies that the pro- $p$ completion $\widehat{G}_{p}$ of an abstract orientable $\mathrm{PD}_{4}$ group $G$ of Euler characteristic 0 is an orientable pro- $p \mathrm{PD}_{4}$ group of Euler characteristic 0 if and only if $G$ is $p$-good (see Corollary $2 c$ )).

It would be interesting to find out whether this generalizes to any dimension, i.e., whether for $G$ an abstract orientable $\mathrm{PD}_{n}$ group of Euler characteristic 0 the pro- $p$ completion $\widehat{G}_{p}$ is an orientable pro- $p \mathrm{PD}_{n}$ group of Euler characteristic 0 if and only if $G$ is $p$-good.

In section 4 we show that when pro- $p$ completions are considered the first of the conditions of Theorem 1 can be substituted with $\widehat{G}_{p}$ is not virtually procyclic. The new ingredient in the proofs of the following theorems is the application of some results 
about virtually Poincaré duality pro- $p$ groups and the number of higher dimensional ends of a pro- $p$ group [7], [8].

Theorem 2. Let $p$ be a prime number and $G$ be an abstract orientable Poincaré $d u$ ality group of dimension 4 and Euler characteristic $\chi(G)=0$ with pro-p completion $\widehat{G}_{p}$. Let $\mathcal{C}$ be a directed set of normal subgroups of p-power index in $G$ such that $\mathcal{C}$ induces the pro- $p$ topology of $G$.

Then $\widehat{G}_{p}$ is an orientable pro-p Poincaré duality group of dimension 4 with Euler characteristic $\chi\left(\widehat{G}_{p}\right)=0$ if and only if all of the following conditions hold:

a) $\widehat{G}_{p}$ is not virtually procyclic;

b) for every $U \in \mathcal{C}$ we have $\sum_{0 \leq i \leq 4}(-1)^{i} \operatorname{dim}_{\mathbb{F}_{p}} H_{i}\left(\hat{U}_{p}, \mathbb{F}_{p}\right)=0$;

c) for every $U \in \mathcal{C}$ we have $2 \operatorname{dim}_{\mathbb{F}_{p}} H_{1}\left(\widehat{U}_{p}, \mathbb{F}_{p}\right)-\operatorname{dim}_{\mathbb{F}_{p}} H_{2}\left(\widehat{U}_{p}, \mathbb{F}_{p}\right)=2$.

Finally we show that if condition c) from Theorem 1 is slightly modified then the only possibility for the pro- $p$ completion of $G$ that is not an orientable PD $_{4}$ pro- $p$ group is to be virtually $\mathbb{Z}_{p}$-by- $\mathbb{Z}_{p}$.

Theorem 3. Let $p$ be a prime number and $G$ be an abstract orientable Poincaré duality group of dimension 4 and Euler characteristic $\chi(G)=0$ with pro- $p$ completion $\widehat{G}_{p}$. Let $\mathcal{C}$ be a directed set of normal subgroups of p-power index in $G$ such that $\mathcal{C}$ induces the pro-p topology of G. Suppose that:

a) $\widehat{G}_{p}$ is not virtually procyclic and is not an orientable pro-p Poincaré duality group of dimension 4;

b) for every $U \in \mathcal{C}$ we have $\sum_{0 \leq i \leq 4}(-1)^{i} \operatorname{dim}_{\mathbb{F}_{p}} H_{i}\left(\widehat{U}_{p}, \mathbb{F}_{p}\right)=0$;

c) $\sup _{U \in \mathcal{C}}\left(2 \operatorname{dim}_{\mathbb{F}_{p}} H_{1}\left(\widehat{U}_{p}, \mathbb{F}_{p}\right)-\operatorname{dim}_{\mathbb{F}_{p}} H_{2}\left(\widehat{U}_{p}, \mathbb{F}_{p}\right)\right)=m<\infty$.

Then $\widehat{G}_{p}$ is virtually $\mathbb{Z}_{p}-b y-\mathbb{Z}_{p}$.

In [5], examples of orientable $\mathrm{PD}_{3}$ groups $M$ with pro- $p$ completion $\widehat{M}_{p}$ procyclic (both cases of finite or infinite occur) were constructed. Then the group $G=\mathbb{Z} \times M$ is an orientable $\mathrm{PD}_{4}$ group with $\chi(G)=0$ and the pro- $p$ completion $\widehat{G}_{p}$ is either virtually $\mathbb{Z}_{p}$ or $\mathbb{Z}_{p}$-by- $\mathbb{Z}_{p}$. The group $M$ is a double of a knot group and so $M$ and $G$ are not soluble, though in both cases $\widehat{G}_{p}$ is soluble.

\section{Preliminaries on abstract and profinite Poincaré duality groups}

1.1. Basic definitions and properties. Let $G$ be an abstract group and $S$ be a commutative ring. A $S[G]$-module $V$ is of type $\mathrm{FP}_{m}$ for some $0 \leq m \leq \infty$ if there exists a projective $S[G]$-resolution of $V$

$$
\mathcal{R}: \cdots \rightarrow R_{i} \rightarrow R_{i-1} \rightarrow \cdots \rightarrow R_{0} \rightarrow V \rightarrow 0,
$$


with $R_{i}$ finitely generated for $i \leq m$. The group $G$ is said to be of type $\mathrm{FP}_{m}$ if the trivial $\mathbb{Z}[G]$-module $\mathbb{Z}$ is of type $\mathrm{FP}_{m}$.

For a profinite group $H$, a profinite ring $R$ and a profinite $R[[H]]$-module $W$ we say that $W$ is of type $\mathrm{FP}_{m}$ over $R$ if there is a profinite projective $R[[H]]$ - resolution of $W$

$$
\mathcal{Q}: \cdots \rightarrow Q_{i} \rightarrow Q_{i-1} \rightarrow \cdots \rightarrow Q_{0} \rightarrow W \rightarrow 0,
$$

with $Q_{i}$ finitely generated for $i \leq m$. The profinite group $H$ is of homological type $\mathrm{FP}_{m}$ over $R$ if the trivial $R[[H]]$-module $R$ is of type $\mathrm{FP}_{m}$.

An abstract group $G$ is a Poincaré duality group of dimension $n$, provided that $G$ is a group of cohomological dimension $\operatorname{cd}(G)=n$ of type $\mathrm{FP}_{\infty}$ and $H^{*}(G, \mathbb{Z}[G])=$ $\operatorname{Ext}_{\mathbb{Z}[G]}^{*}(\mathbb{Z}, \mathbb{Z}[G])$ is concentrated in dimension $n$, where it is $\mathbb{Z}$. If the $G$-action on $H^{n}(G, \mathbb{Z}[G])$ is the trivial one, $G$ is orientable; otherwise $G$ is called non-orientable. There is an equivalent definition of abstract Poincaré duality group of dimension $n$, i.e., there is an isomorphism $H^{i}(G, M) \simeq H_{n-i}\left(G, D \otimes_{\mathbb{Z}} M\right)$ for all $G$-modules $M$ and all $i$, where the dualizing module $D$ is $H^{n}(G, \mathbb{Z}[G]) \simeq \mathbb{Z}$ [2], Ch. 8, Prop. 10.1.

There are two definitions of a profinite Poincaré duality group $H$ of dimension $n$ at a prime $p$ [10], 3.4.6, [15]. The definitions differ at the point whether $H$ should be of type $\mathrm{FP}_{\infty}$ over $\mathbb{Z}_{p}$. As mentioned in the introduction, we call the groups satisfying the definition of [15] strong profinite $\mathrm{PD}_{n}$ groups at $p$ and the groups satisfying the original Tate's definition [10], 3.4.6, [14] we call profinite $\mathrm{PD}_{n}$ groups at $p$. A strong $\mathrm{PD}_{n}$ group at $p$ has cohomological $p$-dimension $\operatorname{cd}_{p}(H)=n$, has type $\mathrm{FP}_{\infty}$ over $\mathbb{Z}_{p}$ and $H^{k}\left(H, \mathbb{Z}_{p}[[H]]\right)=\operatorname{Ext}_{\mathbb{Z}_{p}[[H]]}^{k}\left(\mathbb{Z}_{p}, \mathbb{Z}_{p}[[H]]\right)$ is 0 for $k \neq n$ and is $\mathbb{Z}_{p}$ for $k=n$. If the action of $H$ on $H^{n}\left(H, \mathbb{Z}_{p}[[H]]\right)$ is trivial $H$ is called orientable.

By [15], strong profinite $\mathrm{PD}_{n}$ groups at $p$ are profinite $\mathrm{PD}_{n}$ groups at $p$. For a profinite $\mathrm{PD}_{n}$ group $H$ at $p$ and $A$ an arbitrary $p$-primary finite discrete $H$-module the groups $H^{i}(H, A)$ are finite for all $i$ [10], 3.4.6, [14]. The precise definition of a profinite $\mathrm{PD}_{n}$ group $H$ at $p$ can be found in [10], Chapter 3. Some important properties of such a group $H$ are $\operatorname{cd}_{p}(H)=n$ and $\operatorname{dim}_{\mathbb{F}_{p}} H^{i}\left(H, \mathbb{F}_{p}\right)=\operatorname{dim}_{\mathbb{F}_{p}} H^{n-i}\left(H, \mathbb{F}_{p}\right)$ for all $0 \leq i \leq n$. A profinite $\mathrm{PD}_{n}$ group $H$ at $p$ is a strong profinite $\mathrm{PD}_{n}$ group at $p$ if it is of type $\mathrm{FP}_{\infty}$ over $\mathbb{Z}_{p}$. In [6] the definition of strong profinite $\mathrm{PD}_{n}$ groups at $p$ was adopted (though the name strong was not used). Note that pro- $p \mathrm{PD}_{n}$ groups are always of type $\mathrm{FP}_{\infty}$ over $\mathbb{Z}_{p}$ and over $\mathbb{F}_{p}$, hence are strong pro- $p \mathrm{PD}_{n}$ groups.

Let $G$ be an abstract group of finite cohomological dimension and of type $\mathrm{FP}_{\infty}$. The Euler characteristic $\chi(G)$ as defined in [2], Ch. IX, Sec. 6, is

$$
\begin{aligned}
\sum_{i}(-1)^{i} \mathrm{rk}_{\mathbb{Z}} H_{i}(G, \mathbb{Z}) & =\sum_{i}(-1)^{i} \operatorname{dim}_{\mathbb{F}_{p}} H_{i}\left(G, \mathbb{F}_{p}\right) \\
& =\sum_{i}(-1)^{i} \operatorname{dim}_{\mathbb{F}_{p}} H^{i}\left(G, \mathbb{F}_{p}\right) .
\end{aligned}
$$

If $U$ is a subgroup of finite index in $G$ by [2], Ch. 9, Thm. 6.3, $\chi(U)=(G: U) \chi(G)$. For a profinite group $H$ of finite cohomological $p$-dimension $\operatorname{cd}_{p}(H)$ and type 
$\mathrm{FP}_{\infty}$ over $\mathbb{Z}_{p}$ the Euler $p$-characteristic $\chi_{p}(H)$ of $H$ is

$$
\begin{aligned}
\sum_{i}(-1)^{i} \operatorname{rk}_{\mathbb{Z}_{p}} H_{i}\left(H, \mathbb{Z}_{p}\right) & =\sum_{i}(-1)^{i} \operatorname{dim}_{\mathbb{F}_{p}} H_{i}\left(H, \mathbb{F}_{p}\right) \\
& =\sum_{i}(-1)^{i} \operatorname{dim}_{\mathbb{F}_{p}} H^{i}\left(H, \mathbb{F}_{p}\right),
\end{aligned}
$$

where $H_{i}(H, \cdot)$ and $H^{i}(H, \cdot)$ are the continuous homology and cohomology.

1.2. Korenev's results. Recently more homological properties of pro- $p \mathrm{PD}_{n}$ groups were discovered in [7] and [8]. As shown in [8], if a pro- $p$ group $H$ of type $\mathrm{FP}_{n}$ over $\mathbb{F}_{p}$ has the property that $H^{i}\left(H, \mathbb{F}_{p}[[H]]\right)=0$ for all $0 \leq i<n$ and $0<\operatorname{dim}_{\mathbb{F}_{p}} H^{n}\left(H, \mathbb{F}_{p}[[H]]\right)<\infty$, then $H$ is virtually a pro- $p \mathrm{PD}_{n}$ group. In particular, $\operatorname{dim}_{\mathbb{F}_{p}} H^{n}\left(H, \mathbb{F}_{p}[[H]]\right)=1$ and $H$ is of type $\mathrm{FP}_{\infty}$. An earlier version of the above result was proved in [7], where the case $n=1$ was considered.

Note that for pro- $p$ groups it is still not known whether Stalling's type theorem holds, i.e., if $H$ is a pro- $p$ group with $\operatorname{dim}_{\mathbb{F}_{p}} H^{1}\left(H, \mathbb{F}_{p}[[H]]\right)>0$, then $H$ splits as a free product with amalgamation or an HNN extension over a finite subgroup.

\section{Profinite completions of abstract Poincaré duality groups}

Let $G$ be an abstract group of type $\mathrm{FP}_{\infty}$ and of finite cohomological dimension and let

$$
\mathcal{R}: 0 \rightarrow R_{m} \stackrel{\partial_{m}}{\longrightarrow} R_{m-1} \stackrel{\partial_{m-1}}{\longrightarrow} \cdots \stackrel{\partial_{2}}{\longrightarrow} R_{1} \stackrel{\partial_{1}}{\longrightarrow} R_{0} \stackrel{\partial_{0}}{\rightarrow} \mathbb{Z} \rightarrow 0
$$

be a projective resolution of the trivial (right) $\mathbb{Z}[G]$-module $\mathbb{Z}$ with all projectives finitely generated. Let $\mathcal{C}$ be a directed set of normal subgroups of finite index in $G$, i.e., for $U_{1}, U_{2} \in \mathcal{C}$ there is $U \in \mathcal{C}$ such that $U \subseteq U_{1} \cap U_{2}$. Define

$$
\widehat{G}_{e}=\lim _{U \in C} G / U
$$

and for $U \in \mathcal{C}$ we define $\hat{U}_{\mathcal{C}}$ as the inverse limit $U / M$ over those $M \in \mathcal{C}$ such that $M \subseteq U$.

Consider the complex $\mathcal{R}_{U}=\mathcal{R} \otimes_{\mathbb{Z}[U]} \mathbb{F}_{p}$ for $U \in \mathcal{C}$. Let

$$
\widehat{\mathcal{R}}: 0 \rightarrow \hat{R}_{m} \stackrel{\hat{\partial}_{m}}{\longrightarrow} \hat{R}_{m-1} \stackrel{\hat{\partial}_{m-1}}{\longrightarrow} \cdots \stackrel{\hat{\partial}_{2}}{\longrightarrow} \hat{R}_{1} \stackrel{\hat{\partial}_{1}}{\rightarrow} \hat{R}_{0} \stackrel{\hat{\partial}_{0}}{\rightarrow} \mathbb{Z} \rightarrow 0
$$

be the inverse limit of the complexes $\mathcal{R}_{U}$ over $U \in \mathcal{C}$. Thus by [6], (1),

$$
\widehat{\mathcal{R}} \simeq \mathcal{R} \otimes_{\mathbb{Z}[G]} \mathbb{F}_{p}\left[\left[\hat{G}_{e}\right]\right]
$$

and by [6], Lemma 2.1,

$$
H_{i}(\widehat{\mathcal{R}}) \simeq \lim _{U \in \mathcal{C}} H_{i}\left(\mathcal{R}_{U}\right) \simeq \lim _{U \in \mathcal{C}} H_{i}\left(U, \mathbb{F}_{p}\right)
$$

In the following lemma Tor denotes the left derived functor of $\otimes$ in the category of abstract modules. 
Lemma 1 ([6], Thm. 2.5). Suppose that $G$ is an abstract group of type $\mathrm{FP}_{\infty}$ and finite cohomological dimension, $C$ a directed set of normal subgroups $U$ of finite index in $G$. Suppose further that for a fixed prime $p$ and for all $i \geq 1$,

$$
\lim _{U \in \mathcal{C}} H_{i}\left(U, \mathbb{F}_{p}\right)=0
$$

Then

$$
\operatorname{Tor}_{i}^{\mathbb{Z}[G]}\left(\mathbb{Z},\left(\mathbb{Z} / p^{m} \mathbb{Z}\right)\left[\left[\widehat{G}_{\ell}\right]\right]\right)=0 \quad \text { and } \quad \operatorname{Tor}_{i}^{\mathbb{Z}[G]}\left(\mathbb{Z}, \mathbb{Z}_{p}\left[\left[\widehat{G}_{e}\right]\right]\right)=0
$$

for all $m \geq 1$ and $i \geq 1$. In particular $\widehat{G}_{e}$ is of type $\mathrm{FP}_{\infty}$ over $\mathbb{Z}_{p}$.

Lemma 2 ([6], Cor. 2.7). Suppose that $G$ is an abstract group of finite cohomological dimension $\operatorname{cd}(G)=m$ and type $\mathrm{FP}_{\infty}$. Let $C$ be a directed set of normal subgroups $U$ of finite index in $G$. Suppose further that

$$
\lim _{U \in \mathcal{C}} H_{i}\left(U, \mathbb{F}_{p}\right)=0
$$

for a fixed prime $p$ and for all $1 \leq i \leq m$.

Then the profinite group $\widehat{G}_{\mathcal{E}}$ is of finite cohomological p-dimension $\operatorname{cd}_{p}\left(\widehat{G}_{e}\right) \leq$ m. Further, it is of type $\mathrm{FP}_{\infty}$ over $\mathbb{F}_{p}$ and over $\mathbb{Z}_{p}$, and its Euler $p$-characteristic $\chi_{p}\left(\widehat{G}_{e}\right)=\chi(G)$.

Theorem 4. Let $G$ be an abstract Poincaré duality group of dimension $m$ and let $Y$ be a directed set of normal subgroups of finite index in G. Suppose further that there is a subgroup $G_{0}$ of finite index in $G$ such that $G_{0}$ is orientable, that there is some $U_{0} \in \bigodot^{\text {with }} U_{0} \subseteq G_{0}$ and that, for all $i \geq 1$,

$$
\lim _{U \in \mathcal{C}} H_{i}\left(U, \mathbb{F}_{p}\right)=0
$$

Then $\widehat{G}_{e}$ is a strong profinite Poincaré duality group of dimension $m$ at $p,\left(\widehat{G_{0}}\right) \mathcal{e}$ is a strong orientable profinite Poincaré duality group of dimension $m$ at $p$ and $\chi_{p}\left(\widehat{G}_{e}\right)=\chi(G)$.

Proof. Let

$$
\mathcal{R}: 0 \rightarrow R_{m} \stackrel{\partial_{m}}{\longrightarrow} R_{m-1} \stackrel{\partial_{m-1}}{\longrightarrow} \cdots \stackrel{\partial_{2}}{\rightarrow} R_{1} \stackrel{\partial_{1}}{\rightarrow} R_{0} \stackrel{\partial_{0}}{\rightarrow} \mathbb{Z} \rightarrow 0
$$

be a projective resolution of the trivial $\mathbb{Z}\left[G_{0}\right]$-module $\mathbb{Z}$ with all projectives finitely generated.

Then $H^{i}(S)=H^{i}\left(G_{0}, \mathbb{Z}\left[G_{0}\right]\right)$ is 0 for $i \neq m$ and $\mathbb{Z}$ for $i=m$, where $S=$ $\operatorname{Hom}_{\mathbb{Z}\left[G_{0}\right]}\left(\mathcal{R}^{\mathrm{del}}, \mathbb{Z}\left[G_{0}\right]\right)$ is the dual complex. Thus $S$ is a complex of left $\mathbb{Z}\left[G_{0}\right]$ modules. Define $\mathcal{T}$ the complex obtained from $S$ by adding its unique non-trivial cohomology

$$
\mathcal{T}: 0 \rightarrow S^{0} \rightarrow S^{1} \rightarrow S^{2} \rightarrow \cdots \rightarrow S^{m} \rightarrow H^{m}(S)=\mathbb{Z} \rightarrow 0 .
$$


In particular, the complex $\mathcal{T}$ is a projective resolution of the trivial left $\mathbb{Z}\left[G_{0}\right]$ module $\mathbb{Z}$. By Lemma 1 , $\operatorname{Tor}_{i}^{\mathbb{Z}\left[G_{0}\right]}\left(\mathbb{Z}, \mathbb{Z}_{p}\left[\left[\left(\widehat{G_{0}}\right)_{e}\right]\right]\right)=0$ and similarly we get that $\operatorname{Tor}_{i}^{\mathbb{Z}\left[G_{0}\right]}\left(\mathbb{Z}_{p}\left[\left[\left(\widehat{G_{0}}\right)_{e}\right]\right], \mathbb{Z}\right)=0$ for $i \geq 1$. Thus

$$
\hat{\mathcal{T}}=\mathbb{Z}_{p}\left[\left[\left(\widehat{G_{0}}\right) e\right]\right] \otimes_{\mathbb{Z}\left[G_{0}\right]} \mathcal{T}: 0 \rightarrow T^{0} \rightarrow T^{1} \rightarrow T^{2} \rightarrow \cdots \rightarrow T^{m} \rightarrow \mathbb{Z}_{p} \rightarrow 0
$$

is a projective resolution of the trivial abstract left $\mathbb{Z}_{p}\left[\left[\left(\widehat{G_{0}}\right) e\right]\right]-$ module $\mathbb{Z}_{p}$ with all projectives finitely generated, and hence is a profinite projective resolution of $\mathbb{Z}_{p}$ over $\mathbb{Z}_{p}\left[\left[\left(\widehat{G}_{0}\right) e\right]\right]$.

Let $\hat{\mathcal{T}}^{\text {del }}$ be the complex obtained from $\hat{\mathcal{T}}$ by deleting the term $\mathbb{Z}_{p}$.

Note that $\hat{\mathcal{J}}^{\text {del }}$ is obtained from the complex $\mathcal{R}^{\text {del }}$ of projective finitely generated $\mathbb{Z}\left[G_{0}\right]$-modules by applying first the functor $\operatorname{Hom}_{\mathbb{Z}\left[G_{0}\right]}\left(\cdot, \mathbb{Z}\left[G_{0}\right]\right)$ and then the functor $\mathbb{Z}_{p}\left[\left[\left(\widehat{G_{0}}\right)_{e}\right]\right] \otimes_{\mathbb{Z}\left[G_{0}\right]}$. The composition of these functors is the same as the composition of the functor $\otimes_{\mathbb{Z}\left[G_{0}\right]} \mathbb{Z}_{p}\left[\left[\left(\widehat{G_{0}}\right) \varphi\right]\right]$ and the functor $\operatorname{Hom}_{\mathbb{Z}_{p}\left[\left[\left(\widehat{G_{0}}\right)_{e}\right]\right]}\left(\cdot, \mathbb{Z}_{p}\left[\left[\left(\widehat{G_{0}}\right)_{e}\right]\right]\right)$ if applied on a complex of finitely generated, projective $\mathbb{Z}\left[G_{0}\right]$-modules. Thus

$$
\hat{\mathcal{T}}^{\mathrm{del}} \simeq \operatorname{Hom}_{\mathbb{Z}_{p}\left[\left[\left(\widehat{G_{0}}\right)_{e}\right]\right]}\left(\mathcal{P}^{\mathrm{del}}, \mathbb{Z}_{p}\left[\left[\left(\widehat{G_{0}}\right) \varphi\right]\right]\right),
$$

where $\mathcal{P}=\mathcal{R} \otimes_{\mathbb{Z}\left[G_{0}\right]} \mathbb{Z}_{p}\left[\left[\left(\widehat{G_{0}}\right)_{\ell}\right]\right] \simeq \widehat{\mathcal{R}}$ is an exact complex of right $\mathbb{Z}_{p}\left[\left[\left(\widehat{G_{0}}\right)_{\ell}\right]\right]-$ modules by Lemma 1 . Then

$$
\begin{aligned}
& H^{i}\left(\left(\widehat{G_{0}}\right)_{e}, \mathbb{Z}_{p}\left[\left[\left(\widehat{G_{0}}\right) e\right]\right]\right)=\operatorname{Ext}_{\mathbb{Z}_{p}\left[\left[\left(\widehat{G_{0}}\right)_{e}\right]\right]}\left(\mathbb{Z}_{p}, \mathbb{Z}_{p}\left[\left[\left(\widehat{G_{0}}\right)_{e}\right]\right]\right) \\
& \simeq H^{i}\left(\operatorname{Hom}_{\left.\mathbb{Z}_{p}\left[\left(\widehat{G_{0}}\right)_{e}\right]\right]}\left(\mathcal{P}^{\text {del }}, \mathbb{Z}_{p}\left[\left[\left(\widehat{G_{0}}\right)_{e}\right]\right]\right)\right) \simeq H^{i}\left(\hat{\mathcal{T}}^{\text {del }}\right)
\end{aligned}
$$

is 0 for $i \neq m$ and is $\mathbb{Z}_{p}$ otherwise. Thus $\left(\widehat{G_{0}}\right) \mathcal{e}$ is a strong profinite $\mathrm{PD}_{m}$ group at $p$ and is orientable since in the complex $\hat{\mathcal{T}}$ the module $\mathbb{Z}_{p}$ is the trivial one, i.e., $\left(\widehat{G_{0}}\right)$ e acts trivially on $\mathbb{Z}_{p}$.

Note that $\left(\widehat{G_{0}}\right) e$ is a subgroup of finite index in $\widehat{G} e$ and, by Lemma $2, \widehat{G} e$ is $\mathrm{FP}_{\infty}$ over $\mathbb{Z}_{p}$ and $\operatorname{cd}_{p}\left(\widehat{G}_{e}\right) \leq m$. By [15], 4.2.9,

$$
H^{i}\left(\left(\widehat{G_{0}}\right)_{e}, \mathbb{Z}_{p}\left[\left[\left(\widehat{G_{0}}\right)_{e}\right]\right]\right) \simeq H^{i}\left(\widehat{G_{e}}, \mathbb{Z}_{p}\left[\left[\widehat{G}_{e}\right]\right]\right) .
$$

Then $H^{*}\left(\hat{G}_{\mathcal{C}}, \mathbb{Z}_{p}\left[\left[\hat{G}_{e}\right]\right]\right)$ is concentrated in dimension $m$, where it is $\mathbb{Z}_{p}$, so $\hat{G}_{\mathcal{C}}$ is a strong profinite $\mathrm{PD}_{m}$ group at $p$.

\section{Profinite completions of Poincaré duality groups of dimension 4 and Euler characteristic 0}

Lemma 3. Let $G$ be an abstract orientable Poincaré duality group of dimension 4 and Euler characteristic $\chi(G)=0$. Then

$$
\begin{aligned}
& 2 \operatorname{dim}_{\mathbb{F}_{p}} H_{1}\left(G, \mathbb{F}_{p}\right)-\operatorname{dim}_{\mathbb{F}_{p}} H_{2}\left(G, \mathbb{F}_{p}\right) \\
& \quad=2=2 \operatorname{dim}_{\mathbb{F}_{p}} H^{1}\left(G, \mathbb{F}_{p}\right)-\operatorname{dim}_{\mathbb{F}_{p}} H^{2}\left(G, \mathbb{F}_{p}\right) .
\end{aligned}
$$


Proof. Indeed $\chi(G)=0$ together with $H_{4-i}\left(G, \mathbb{F}_{p}\right) \simeq H^{i}\left(G, \mathbb{F}_{p}\right) \simeq H_{i}\left(G, \mathbb{F}_{p}\right)$ for $i=0$ and $i=1$ gives

$$
\begin{aligned}
0=\chi(G)= & \sum_{0 \leq i \leq 4}(-1)^{i} \operatorname{dim}_{\mathbb{F}_{p}} H_{i}\left(G, \mathbb{F}_{p}\right) \\
= & 1-\operatorname{dim}_{\mathbb{F}_{p}} H_{1}\left(G, \mathbb{F}_{p}\right)+\operatorname{dim}_{\mathbb{F}_{p}} H_{2}\left(G, \mathbb{F}_{p}\right) \\
& \quad-\operatorname{dim}_{\mathbb{F}_{p}} H^{1}\left(G, \mathbb{F}_{p}\right)+\operatorname{dim}_{\mathbb{F}_{p}} H^{0}\left(G, \mathbb{F}_{p}\right) \\
= & 2-2 \operatorname{dim}_{\mathbb{F}_{p}} H_{1}\left(G, \mathbb{F}_{p}\right)+\operatorname{dim}_{\mathbb{F}_{p}} H_{2}\left(G, \mathbb{F}_{p}\right) .
\end{aligned}
$$

The proof is completed by the isomorphisms $H^{1}\left(G, \mathbb{F}_{p}\right) \simeq G /[G, G] G^{p} \simeq$ $H_{1}\left(G, \mathbb{F}_{p}\right)$ and $H^{2}\left(G, \mathbb{F}_{p}\right) \simeq H_{2}\left(G, \mathbb{F}_{p}\right)$.

Lemma 4. Let $H$ be a profinite orientable Poincaré duality group of dimension 4 at $p$ with Euler p-characteristic $\chi_{p}(H)=0$.

Then

$$
\begin{aligned}
& 2 \operatorname{dim}_{\mathbb{F}_{p}} H_{1}\left(H, \mathbb{F}_{p}\right)-\operatorname{dim}_{\mathbb{F}_{p}} H_{2}\left(H, \mathbb{F}_{p}\right) \\
& \quad=2=2 \operatorname{dim}_{\mathbb{F}_{p}} H^{1}\left(H, \mathbb{F}_{p}\right)-\operatorname{dim}_{\mathbb{F}_{p}} H^{2}\left(H, \mathbb{F}_{p}\right) .
\end{aligned}
$$

Proof. Note that for $0 \leq i \leq 4$ we have $\operatorname{dim}_{\mathbb{F}_{p}} H_{i}\left(H, \mathbb{F}_{p}\right)=\operatorname{dim}_{\mathbb{F}_{p}} H^{i}\left(H, \mathbb{F}_{p}\right)$ by Pontryagin duality and $\operatorname{dim}_{\mathbb{F}_{p}} H^{i}\left(H, \mathbb{F}_{p}\right)=\operatorname{dim}_{\mathbb{F}_{p}} H^{4-i}\left(H, \mathbb{F}_{p}\right)$ by Tate's definition of Poincaré duality. Then the proof is completed as the proof of Lemma 3.

Proof of Theorem 1. Suppose now that the conditions a), b) and c) hold.

Since $G$ is an abstract orientable $\mathrm{PD}_{4}$ group every subgroup of finite index in $G$ is an abstract orientable $\mathrm{PD}_{4}$ group. In particular this holds for any $U \in \mathcal{C}$ and we have $H_{4}\left(U, \mathbb{F}_{p}\right) \simeq H^{0}\left(U, \mathbb{F}_{p}\right) \simeq \mathbb{F}_{p}$. Then the inverse limit of $H_{4}\left(U, \mathbb{F}_{p}\right)$ over $U \in \mathcal{C}$ is either $\mathbb{F}_{p}$ or 0 . It cannot be $\mathbb{F}_{p}$ otherwise there exists an ideal in $\mathbb{F}_{p}\left[\left[\widehat{G}_{\ell}\right]\right]$ isomorphic to $\mathbb{F}_{p}$ and this easily contradicts the fact that $\hat{G}_{e}$ has an infinite Sylow $p$-subgroup (note that $\operatorname{cd}_{p}(H)=\operatorname{cd}_{p}\left(\widehat{G}_{\ell}\right) \leq 4<\infty$ for $H$ a Sylow $p$-subgroup of $\left.\widehat{G}_{\ell}\right)$. Indeed if the inverse limit is $\mathbb{F}_{p}$ by (3) $H_{4}(\widehat{\mathcal{R}}) \simeq \mathbb{F}_{p}$ and by going down to a subgroup of finite index if necessary, we can assume that $\widehat{G}_{e}$ acts trivially on $H_{4}(\widehat{\mathcal{R}}) \subseteq \widehat{R}_{4}$. Note that $\widehat{R}_{4}$ is a finite rank projective $\mathbb{F}_{p}\left[\left[\widehat{G}_{\ell}\right]\right]$-module, hence a direct summand of the finite rank free $\mathbb{F}_{p}\left[\left[\widehat{G}_{e}\right]\right]$-module $F$. Thus the trivial $\mathbb{F}_{p}\left[\left[\widehat{G}_{e}\right]\right]$-module $\mathbb{F}_{p}$ is a submodule of $F$ and projecting to one of the free factors $\mathbb{F}_{p}\left[\left[\hat{G}_{e}\right]\right]$ of $F$, we see that $\mathbb{F}_{p}\left[\left[\widehat{G}_{e}\right]\right]$ contains the trivial $\mathbb{F}_{p}\left[\left[\widehat{G}_{e}\right]\right]-$ module $\mathbb{F}_{p}$ as a submodule, a contradiction. A different argument using restriction and corestriction can be used as in the proof of [6], Prop. 3.1.

Note that we have shown that

$$
H_{4}(\widehat{\mathcal{R}}) \simeq \lim _{U \in \mathcal{C}} H_{4}\left(U, \mathbb{F}_{p}\right)=0 .
$$

As tensor product is a right exact functor $H_{0}(\widehat{\mathcal{R}})=0$. The condition that $\mathcal{T}$ is subgroup, extension and quotient closed and contains the cyclic group with $p$ 
elements implies that $\mathcal{T}$ contains all finite $p$-groups. Then, since $\mathcal{C}$ induces the pro$\mathcal{T}$ topology of $G$, we obtain that for every $U \in \mathcal{C}$ there is a subgroup $U_{1} \in \mathcal{C}$ with $U_{1} \subseteq[U, U] U^{p}$. Hence the canonical map

$$
\varphi_{1, U}: H_{1}\left(U, \mathbb{F}_{p}\right) \rightarrow H_{1}\left(\hat{U}_{e}, \mathbb{F}_{p}\right)
$$

is an isomorphism and

$$
\begin{aligned}
H_{1}(\widehat{\mathcal{R}}) & \simeq \lim _{U \in \mathcal{C}} H_{1}\left(U, \mathbb{F}_{p}\right) \simeq \lim _{U \in \mathcal{C}} H_{1}\left(\hat{U}_{\mathcal{C}}, \mathbb{F}_{p}\right) \\
& =H_{1}\left(\stackrel{\lim }{\leftarrow} U \in \mathcal{U}_{\mathcal{U}}, \mathbb{F}_{p}\right)=H_{1}\left(1, \mathbb{F}_{p}\right)=0 .
\end{aligned}
$$

We claim that the canonical map

$$
\varphi_{2, U}: H_{2}\left(U, \mathbb{F}_{p}\right) \rightarrow H_{2}\left(\widehat{U}_{\mathcal{C}}, \mathbb{F}_{p}\right)
$$

is an isomorphism.

Indeed $H_{2}\left(U, \mathbb{F}_{p}\right) \simeq H_{2}\left(\mathcal{R} \otimes_{\mathbb{Z}[U]} \mathbb{F}_{p}\right) \simeq H_{2}\left(\widehat{\mathcal{R}} \otimes_{\mathbb{F}_{p}\left[\left[\hat{U}_{\mathcal{C}}\right]\right.} \mathbb{F}_{p}\right)$. The partial profinite projective $\mathbb{F}_{p}\left[\left[\hat{U}_{e}\right]\right]$-resolution $\hat{R}_{2} \stackrel{\hat{\partial}_{2}}{\rightarrow} \widehat{R}_{1} \stackrel{\hat{\partial}_{1}}{\rightarrow} \hat{R}_{0} \rightarrow \mathbb{F}_{p} \rightarrow 0$ of $\mathbb{F}_{p}$ can be extended to a partial profinite projective $\mathbb{F}_{p}\left[\left[\hat{U}_{e}\right]\right]-$-resolution

$$
S: S \stackrel{\nu}{\rightarrow} \hat{R}_{2} \stackrel{\hat{\partial_{2}}}{\rightarrow} \hat{R}_{1} \stackrel{\hat{\partial}_{1}}{\rightarrow} \hat{R}_{0} \rightarrow \mathbb{F}_{p} \rightarrow 0,
$$

where $S$ contains $\hat{R}_{3}$ as a closed submodule and $v$ is an extension of $\hat{\partial}_{3}$ from (2). Thus $H_{2}\left(\hat{U}_{\complement}, \mathbb{F}_{p}\right) \simeq H_{2}\left(\mathcal{S} \otimes_{\mathbb{F}_{p}\left[\left[\hat{U}_{e}\right]\right]} \mathbb{F}_{p}\right)$ is a quotient of $H_{2}\left(\widehat{\mathcal{R}} \otimes_{\mathbb{F}_{p}\left[\left[\hat{U}_{e}\right]\right]} \mathbb{F}_{p}\right)$, and $\varphi_{2, U}$ is surjective.

Then by Lemma 3, Lemma 4 and condition c) of the theorem, it follows that

$$
\begin{aligned}
\operatorname{dim}_{\mathbb{F}_{p}} \operatorname{ker}\left(\varphi_{2, U}\right) & =\operatorname{dim}_{\mathbb{F}_{p}} H_{2}\left(U, \mathbb{F}_{p}\right)-\operatorname{dim}_{\mathbb{F}_{p}} H_{2}\left(\widehat{U}_{\mathcal{C}}, \mathbb{F}_{p}\right) \\
& =\left(2 \operatorname{dim}_{\mathbb{F}_{p}} H_{1}\left(U, \mathbb{F}_{p}\right)-2\right)-\left(2 \operatorname{dim}_{\mathbb{F}_{p}} H_{1}\left(\widehat{U}_{e}, \mathbb{F}_{p}\right)-2\right)=0 .
\end{aligned}
$$

In particular (5) holds and we have

$$
\begin{aligned}
H_{2}(\widehat{\mathcal{R}}) & \simeq \lim _{U \in \mathcal{C}} H_{2}\left(U, \mathbb{F}_{p}\right) \simeq \lim _{U \in \mathcal{U}} H_{2}\left(\hat{U}_{\mathcal{C}}, \mathbb{F}_{p}\right) \\
& =H_{2}\left(\stackrel{\lim }{\longleftarrow} U \in \mathcal{U}_{\mathcal{U}}, \mathbb{F}_{p}\right)=H_{2}\left(1, \mathbb{F}_{p}\right)=0 .
\end{aligned}
$$

Note that we have proved by now that

$$
H_{i}(\widehat{\mathcal{R}})=0 \quad \text { for } i \neq 3 \text {. }
$$

Let

$$
\mathcal{P}: P \stackrel{\mu}{\rightarrow} \hat{R}_{3} \stackrel{\hat{\partial}_{3}}{\rightarrow} \hat{R}_{2} \stackrel{\hat{\partial}_{2}}{\rightarrow} \hat{R}_{1} \stackrel{\hat{\partial}_{1}}{\rightarrow} \hat{R}_{0} \stackrel{\hat{\partial}_{0}}{\rightarrow} \mathbb{F}_{p} \rightarrow 0
$$

be a partial profinite projective resolution of the trivial $\mathbb{F}_{p}\left[\left[\widehat{G}_{\ell}\right]\right]$-module $\mathbb{F}_{p}$ with $\hat{R}_{4}$ a closed submodule of $P$ and $\mu$ an extension of $\hat{\partial}_{4}$. Hence $\mathcal{P}$ is a partial profinite 
projective resolution of the trivial $\mathbb{F}_{p}\left[\left[\hat{U}_{\ell}\right]\right]$-module $\mathbb{F}_{p}$ for every $U \in \mathcal{C}$. Then the natural embedding of the 4-skeleton $\widehat{\mathcal{R}}^{(4)}$ in $\mathcal{P}$ induces an epimorphism

$$
H_{3}\left(U, \mathbb{F}_{p}\right) \simeq H_{3}\left(\widehat{\mathcal{R}} \hat{\otimes}_{\mathbb{F}_{p}\left[\left[\hat{U}_{e}\right]\right]} \mathbb{F}_{p}\right) \rightarrow H_{3}\left(\mathcal{P} \hat{\otimes}_{\mathbb{F}_{p}\left[\left[\hat{U}_{e}\right]\right]} \mathbb{F}_{p}\right) \simeq H_{3}\left(\hat{U}_{\mathcal{C}}, \mathbb{F}_{p}\right) .
$$

Consequently the canonical map

$$
\varphi_{3, U}: H_{3}\left(U, \mathbb{F}_{p}\right) \rightarrow H_{3}\left(\hat{U}_{e}, \mathbb{F}_{p}\right)
$$

is an epimorphism, hence

$$
\operatorname{dim}_{\mathbb{F}_{p}} H_{3}\left(U, \mathbb{F}_{p}\right)-\operatorname{dim}_{\mathbb{F}_{p}} H_{3}\left(\hat{U}_{e}, \mathbb{F}_{p}\right) \geq 0 .
$$

Note that by condition $b$ ),

$$
\begin{aligned}
& \sum_{0 \leq i \leq 4}(-1)^{i} \operatorname{dim}_{\mathbb{F}_{p}} H_{i}\left(\widehat{U}_{\mathcal{C}}, \mathbb{F}_{p}\right) \\
& \quad=0=\chi(G)=\chi(U)=\sum_{0 \leq i \leq 4}(-1)^{i} \operatorname{dim}_{\mathbb{F}_{p}} H_{i}\left(U, \mathbb{F}_{p}\right)
\end{aligned}
$$

and since $\varphi_{i, U}$ is an isomorphism for $i=1,2$ it follows that $\operatorname{dim}_{\mathbb{F}_{p}} H_{i}\left(U, \mathbb{F}_{p}\right)=$ $\operatorname{dim}_{\mathbb{F}_{p}} H_{i}\left(\hat{U}_{e}, \mathbb{F}_{p}\right)$ for $i=1,2$. Then by $H_{4}\left(U, \mathbb{F}_{p}\right) \simeq H^{0}\left(U, \mathbb{F}_{p}\right) \simeq \mathbb{F}_{p},(7)$ and (8) we get

$$
\begin{aligned}
0 & \leq \operatorname{dim}_{\mathbb{F}_{p}} H_{3}\left(U, \mathbb{F}_{p}\right)-\operatorname{dim}_{\mathbb{F}_{p}} H_{3}\left(\hat{U}_{e}, \mathbb{F}_{p}\right) \\
& =\sum_{0 \leq i \neq 3 \leq 4}(-1)^{i}\left(\operatorname{dim}_{\mathbb{F}_{p}} H_{i}\left(U, \mathbb{F}_{p}\right)-\operatorname{dim}_{\mathbb{F}_{p}} H_{i}\left(\hat{U}_{e}, \mathbb{F}_{p}\right)\right) \\
& =\operatorname{dim}_{\mathbb{F}_{p}} H_{4}\left(U, \mathbb{F}_{p}\right)-\operatorname{dim}_{\mathbb{F}_{p}} H_{4}\left(\widehat{U}_{e}, \mathbb{F}_{p}\right) \\
& \leq \operatorname{dim}_{\mathbb{F}_{p}} H_{4}\left(U, \mathbb{F}_{p}\right)=1
\end{aligned}
$$

and so

$$
\operatorname{dim}_{\mathbb{F}_{p}} \operatorname{ker}\left(\varphi_{3, U}\right)=\operatorname{dim}_{\mathbb{F}_{p}} H_{3}\left(U, \mathbb{F}_{p}\right)-\operatorname{dim}_{\mathbb{F}_{p}} H_{3}\left(\widehat{U}_{\complement}, \mathbb{F}_{p}\right) \leq 1 .
$$

Consider the short exact sequence

$$
0 \rightarrow \operatorname{ker}\left(\varphi_{3, U}\right) \rightarrow H_{3}\left(U, \mathbb{F}_{p}\right) \rightarrow H_{3}\left(\hat{U}_{e}, \mathbb{F}_{p}\right) \rightarrow 0
$$

and the corresponding exact sequence

$$
\begin{aligned}
& 0 \rightarrow \lim _{U \in e^{-}} \operatorname{ker}\left(\varphi_{3, U}\right) \rightarrow \lim _{U \in e^{-}} H_{3}\left(U, \mathbb{F}_{p}\right) \\
& \rightarrow \lim _{U \in \mathcal{C}} H_{3}\left(\hat{U}_{\mathcal{C}}, \mathbb{F}_{p}\right) \simeq H_{3}\left(\lim _{U \in \mathcal{C}} \hat{U}_{\mathcal{C}}, \mathbb{F}_{p}\right)=0 \rightarrow \cdots .
\end{aligned}
$$

Then by (9),

$$
H_{3}(\widehat{\mathcal{R}}) \simeq \lim _{U \in \mathcal{C}} H_{3}\left(U, \mathbb{F}_{p}\right) \simeq \lim _{U \in \mathcal{C}} \operatorname{ker}\left(\varphi_{3, U}\right)
$$


is either zero or $\mathbb{F}_{p}$.

Define $V=H_{3}(\widehat{\mathcal{R}})$ and suppose that $V \neq 0$, consequently $V \simeq \mathbb{F}_{p}$. Let $U \in \mathcal{\ell}$ be such that $U$ acts trivially on $V$. We claim that since $2 \leq \operatorname{cd}_{p}\left(\hat{G}_{\ell}\right)=t<\infty$, the projective dimension of $V$ as a profinite $\mathbb{F}_{p}\left[\left[\hat{U}_{e}\right]\right]$-module is $\max \{t-4,0\}$.

Indeed if $0 \rightarrow W_{1} \rightarrow W \rightarrow W_{2} \rightarrow 0$ is a short exact sequence of profinite modules with $W$ projective, then either the projective dimension of $W_{1}$ is the projective dimension of $W_{2}$ minus 1 or $W_{1}$ and $W_{2}$ are projective, i.e., both have projective dimension 0 (this follows from the fact that the projective dimension $k$ of a profinite $\mathbb{F}_{p}\left[\left[\hat{U}_{\mathcal{C}}\right]\right]$-module $M$ is the minimal non-negative integer $k$ such that $\widehat{\operatorname{Ext}}_{\mathbb{F}_{p}\left[\left[\hat{U}_{\mathcal{C}}\right]\right]}^{k+1}(M, S)=0$ for every discrete finite $p$-primary $\mathbb{F}_{p}\left[\left[\widehat{U}_{\mathcal{C}}\right]\right]$-module $S$, where $\widehat{\mathrm{Ext}}$ is the derived functor of continuous Hom). Since the trivial $\mathbb{F}_{p}\left[\left[\widehat{G}_{e}\right]\right]$-module $\mathbb{F}_{p}$ has profinite projective dimension $t$ over $\mathbb{F}_{p}\left[\left[\widehat{G}_{\ell}\right]\right]$, by (6) we get that $\operatorname{ker}\left(\widehat{\partial_{3}}\right)$ has projective dimension $s=\max \{t-4,0\}$ as a profinite $\mathbb{F}_{p}\left[\left[\hat{G}_{\ell}\right]\right]$-module.

Hence $\operatorname{ker}\left(\hat{\partial_{3}}\right)$ has projective dimension $s=\max \{t-4,0\}$ as a profinite $\mathbb{F}_{p}\left[\left[\hat{U}_{\ell}\right]\right]-$ module.

Consider the short exact sequence of profinite $\mathbb{F}_{p}\left[\left[\hat{U}_{\ell}\right]\right]$-modules

$$
\mathcal{A}: 0 \rightarrow A_{1}=\hat{R}_{4} \stackrel{\hat{\partial}_{4}}{\rightarrow} A_{0}=\operatorname{ker}\left(\hat{\partial}_{3}\right) \rightarrow V \rightarrow 0,
$$

where $V \simeq \mathbb{F}_{p}$ is the trivial module. Since $A_{1}$ is projective, for every discrete finite $p$-primary $\mathbb{F}_{p}\left[\left[\hat{U}_{\mathcal{C}}\right]\right]$-module $S$ and $i \geq 2$, there is an isomorphism

$$
\widehat{\operatorname{Ext}}_{\mathbb{F}_{p}\left[\left[\hat{U}_{e}\right]\right]}^{i}(V, S) \simeq \widehat{\operatorname{Ext}}_{\mathbb{F}_{p}\left[\left[\hat{U}_{e}\right]\right]}^{i}\left(A_{0}, S\right) .
$$

In particular if $\widehat{\operatorname{Ext}}_{\mathbb{F}_{p}\left[\left[\hat{U}_{e}\right]\right]}^{i}(V, S) \neq 0$ for some $i \geq 2$ (i.e., $\operatorname{pd}_{\mathbb{F}_{p}\left[\left[\hat{U}_{e}\right]\right]}(V) \geq 2$ ) we get that $\operatorname{pd}_{\mathbb{F}_{p}\left[\left[\hat{U}_{e}\right]\right]}(V)=\operatorname{pd}_{\mathbb{F}_{p}\left[\left[\hat{U}_{e}\right]\right]}\left(A_{0}\right)$. Finally since

$$
\operatorname{pd}_{\mathbb{F}_{p}\left[\left[\hat{U}_{e}\right]\right]}(V)=\operatorname{pd}_{\mathbb{F}_{p}\left[\left[\hat{U}_{e}\right]\right]}\left(\mathbb{F}_{p}\right)=\operatorname{cd}_{p}\left(\hat{U}_{\complement}\right)=\operatorname{cd}_{p}\left(\hat{G}_{\complement}\right)=t \geq 2,
$$

we obtain that

$$
t=\operatorname{pd}_{\mathbb{F}_{p}\left[\left[\hat{U}_{e}\right]\right]}(V)=\operatorname{pd}_{\mathbb{F}_{p}\left[\left[\hat{U}_{e}\right]\right]}\left(A_{0}\right)=s=\max \{t-4,0\}<t,
$$

a contradiction.

Thus

$$
H_{3}(\widehat{\mathcal{R}})=0
$$

and we have shown that

$$
H_{i}(\hat{R})=0 \quad \text { for all } i \geq 1 .
$$

Then by (3) we can apply Theorem 4 to deduce that $\widehat{G}_{e}$ is a strong profinite orientable $\mathrm{PD}_{4}$ group at $p$.

Finally we observe that if $\hat{G}_{\mathcal{C}}$ is a profinite orientable $\mathrm{PD}_{4}$ group at $p$, then obviously all conditions a), b) and c) hold. 
Corollary 1. Let $p$ be a prime number and $G$ be an abstract orientable Poincaré duality group of dimension 4 and Euler characteristic $\chi(G)=0$. Let $\mathcal{T}$ be a class of finite groups closed under subgroups, extensions and quotients, let the cyclic group of order $p$ be in $\mathcal{T}$ and let $\mathcal{C}$ be a directed set of normal subgroups of finite index in $G$ such that $\mathcal{C}$ induces the pro- $\mathcal{T}$ topology of $G$.

Then the following conditions are equivalent:

a) $\widehat{G}_{\mathcal{C}}$ is an orientable profinite Poincaré duality group of dimension 4 at the prime $p$ with Euler $p$-characteristic $\chi_{p}\left(\widehat{G}_{e}\right)=0$

b) $\widehat{G}_{e}$ is a strong orientable profinite Poincaré duality group of dimension 4 at the prime $p$ with Euler $p$-characteristic $\chi_{p}\left(\widehat{G}_{\mathcal{C}}\right)=0$;

c) $\operatorname{Tor}_{i}^{\mathbb{Z}[G]}\left(\mathbb{Z}, \mathbb{F}_{p}\left[\left[\widehat{G}_{e}\right]\right]\right)=0$ for every $i \geq 1$;

d) $\operatorname{Tor}_{i}^{\mathbb{Z}[G]}\left(\mathbb{Z}, \mathbb{Z}_{p}\left[\left[\widehat{G}_{\mathcal{C}}\right]\right]\right)=0$ for every $i \geq 1$.

Proof. By Theorem 1 item a) is equivalent with item b). Using again Theorem 1, $\widehat{G}_{\mathcal{C}}$ is an orientable profinite $\mathrm{PD}_{4}$ group at $p$ with $\chi_{p}\left(\widehat{G}_{\mathcal{C}}\right)=0$ if and only if the conditions a), b) and c) from Theorem 1 hold. The proof of Theorem 1 shows that if these three conditions hold, then $\widehat{\mathcal{R}}$ is an exact complex.

Conversely, if $\widehat{\mathcal{R}}$ is an exact complex, that is,

$$
0=H_{i}(\widehat{\mathcal{R}}) \simeq \lim _{U \in \mathcal{C}} H_{i}\left(U, \mathbb{F}_{p}\right)
$$

for $i \geq 1$, we get by Theorem 4 that $\widehat{G}_{\mathcal{C}}$ is a strong orientable profinite $\mathrm{PD}_{4}$ group at $p$ with $\chi_{p}\left(\widehat{G}_{\mathcal{E}}\right)=0$, hence is a profinite orientable $\mathrm{PD}_{4}$ group at $p$.

Thus item a) is equivalent with $H_{i}(\widehat{\mathcal{R}})=0$ for all $i \geq 1$.

Since $H_{i}(\widehat{\mathcal{R}}) \simeq \operatorname{Tor}_{i}^{\mathbb{Z}[G]}\left(\mathbb{Z}, \mathbb{F}_{p}\left[\left[\widehat{G}_{\mathcal{C}}\right]\right]\right)$ for $i \geq 1$ we see that a) and c) are equivalent. Furthermore, by Lemma 1 , if (11) holds then d) holds, i.e., a) implies d).

If item d) holds then $S=\mathcal{R} \otimes_{\mathbb{Z}[G]} \mathbb{Z}_{p}\left[\left[\widehat{G}_{\mathcal{C}}\right]\right]$ is an abstract projective resolution of $\mathbb{Z}_{p}$ over $\mathbb{Z}_{p}\left[\left[\widehat{G}_{\ell}\right]\right]$ of finite length and finitely generated projectives in any dimension, so $S$ is a profinite projective resolution of $\mathbb{Z}_{p}$ as a profinite $\mathbb{Z}_{p}\left[\left[\widehat{G}_{\mathcal{C}}\right]\right]$-module, hence as a profinite $\mathbb{Z}_{p}$-module.

Since $S \otimes_{\mathbb{Z}_{p}} \mathbb{F}_{p} \simeq S \hat{\otimes}_{\mathbb{Z}_{p}} \mathbb{F}_{p}$ we have

$$
\begin{aligned}
\operatorname{Tor}_{i}^{\mathbb{Z}[G]}\left(\mathbb{Z}, \mathbb{F}_{p}\left[\left[\widehat{G}_{e}\right]\right]\right) & =H_{i}\left(\mathcal{R} \otimes_{\mathbb{Z}[G]} \mathbb{F}_{p}\left[\left[\widehat{G}_{e}\right]\right]\right) \\
& \simeq H_{i}\left(S \otimes_{\mathbb{Z}_{p}} \mathbb{F}_{p}\right) \\
& \simeq H_{i}\left(S \widehat{\otimes}_{\mathbb{Z}_{p}} \mathbb{F}_{p}\right)=\widehat{\operatorname{Tor}}_{i}^{\mathbb{Z}_{p}}\left(\mathbb{Z}_{p}, \mathbb{F}_{p}\right)=0 \quad \text { for } i \geq 1,
\end{aligned}
$$

where Tor denotes the left derived functor of $\widehat{\otimes}$ in the category of profinite modules, i.e., d) implies c).

Corollary 2. Let $p$ be a prime number and $G$ be an abstract orientable Poincaré duality group of dimension 4 and Euler characteristic $\chi(G)=0$. Let $\mathcal{T}$ be a class of 
finite groups closed under subgroups, extensions and quotients, let the cyclic group of order $p$ be in $\mathcal{T}$ and let $\mathcal{C}$ be a directed set of normal subgroups $U$ of finite index in $G$ such that $\mathcal{C}$ induces the pro- $\mathcal{T}$ topology of $G$.

Then for the pro- $\mathcal{T}$ completion $\widehat{G}$ e of $G$ the following results hold:

a) $\widehat{G}_{e}$ is an orientable profinite Poincaré duality group of dimension 4 at p with Euler $p$-characteristic $\chi_{p}\left(\widehat{G}_{e}\right)=0$ if and only if, for every $U \in \mathcal{C}$, the canonical maps between abstract and continuous homology

$$
\varphi_{i, U}: H_{i}\left(U, \mathbb{F}_{p}\right) \rightarrow H_{i}\left(\widehat{U}_{e}, \mathbb{F}_{p}\right)
$$

are isomorphisms for all $i$;

b) $\hat{G}_{e}$ is an orientable Poincaré duality group of dimension 4 at $p$ with Euler $p$ characteristic $\chi_{p}\left(\widehat{G}_{\mathcal{C}}\right)=0$ if and only if, for every $U \in \mathcal{C}$, the canonical maps between continuous and abstract cohomology

$$
\mu_{i, U}: H^{i}\left(\widehat{U}_{e}, \mathbb{F}_{p}\right) \rightarrow H^{i}\left(U, \mathbb{F}_{p}\right)
$$

are isomorphisms for all $i$;

c) the pro-p completion of $G$ is an orientable Poincaré duality pro-p group of dimension 4 and Euler characteristic 0 if and only if $G$ is p-good.

Proof. 1. If $\varphi_{i, U}$ is an isomorphism for every $U \in \mathcal{C}$

$$
\begin{aligned}
\lim _{U \in \mathscr{C}} H_{i}\left(U, \mathbb{F}_{p}\right) & \simeq \lim _{U \in \mathscr{C}} H_{i}\left(\hat{U}_{\mathscr{C}}, \mathbb{F}_{p}\right) \\
& =H_{i}(\underbrace{\lim }_{\widehat{U} \in \mathcal{C}} \hat{U}_{\mathscr{C}}, \mathbb{F}_{p})=H_{i}\left(1, \mathbb{F}_{p}\right)=0 \quad \text { for } i \geq 1,
\end{aligned}
$$

and by Theorem 4, $\widehat{G}_{\mathcal{C}}$ is an orientable profinite $\mathrm{PD}_{4}$ group at $p$.

2. Suppose now that $\hat{G}_{\ell}$ is an orientable profinite $\mathrm{PD}_{4}$ group at $p$ with $\chi_{p}\left(\hat{G}_{\ell}\right)=$ 0 and $\mathcal{R}$ is the complex (1) for $m=4$.

By Corollary $1, \mathcal{R} \otimes_{\mathbb{Z}[G]} \mathbb{Z}_{p}\left[\left[\hat{G}_{e}\right]\right]$ is exact and the same holds for $G$ substituted with any $U \in \mathcal{C}$ and any projective resolution of finite type and length at most 4 of the trivial $\mathbb{Z}[U]$-module $\mathbb{Z}$. In particular, $\mathcal{Q}=\mathcal{R} \otimes_{\mathbb{Z}[U]} \mathbb{Z}_{p}\left[\left[\hat{U}_{\mathcal{C}}\right]\right]$ is exact. We can use the exactness of $\mathcal{Q}$ to show that the natural maps $H_{i}(U, M) \rightarrow H_{i}\left(\hat{U}_{\complement}, M\right)$ and $H^{i}\left(\widehat{U}_{\complement}, M\right) \rightarrow H^{i}(U, M)$ are isomorphisms for every $p$-primary finite discrete $\widehat{G}_{p^{-}}$ module $M$. In particular, $\varphi_{i, U}$ and $\mu_{i, U}$ are isomorphisms. Indeed

$$
H_{i}\left(\hat{U}_{e}, M\right) \simeq H_{i}\left(\mathcal{Q} \widehat{\otimes}_{\mathbb{Z}_{p}\left[\left[\hat{U}_{e}\right]\right]} M\right) \simeq H_{i}\left(\mathcal{R} \otimes_{\mathbb{Z}[U]} M\right) \simeq H_{i}(U, M)
$$

and

$$
H^{i}\left(\widehat{U}_{\mathcal{C}}, M\right) \simeq H^{i}\left(\widehat{\operatorname{Hom}}_{\mathbb{Z}_{p}\left[\left[\hat{U}_{e}\right]\right]}(\mathcal{Q}, M)\right) \simeq H^{i}\left(\operatorname{Hom}_{\mathbb{Z}[U]}(\mathcal{R}, M)\right) \simeq H^{i}(U, M),
$$


where $\widehat{\mathrm{Hom}}$ denotes continuous homomorphisms. In particular, if $\mathcal{T}$ is the class of all finite $p$-groups and $U=G$, then (12) implies that $G$ is $p$-good.

3. Now suppose that $\mu_{i, U}$ is an isomorphism for all $i \geq 1$ and $U \in \mathcal{C}$.

We show that all three conditions a), b) and c) of Theorem 1 hold. Indeed, $H^{5}\left(\widehat{U}_{\mathcal{C}}, \mathbb{F}_{p}\right) \simeq H^{5}\left(U, \mathbb{F}_{p}\right)=0$ for all $U \in \mathcal{C}$ and consequently by [14], Prop. 21', $\operatorname{cd}_{p}\left(\hat{G}_{\ell}\right) \leq 4$. Furthermore $H^{4}\left(\hat{U}_{e}, \mathbb{F}_{p}\right) \simeq H^{4}\left(U, \mathbb{F}_{p}\right) \simeq \mathbb{F}_{p} \neq 0$, in particular $\operatorname{cd}_{p}\left(\hat{U}_{\ell}\right) \geq 4$ and so $4 \leq \operatorname{cd}_{p}\left(\hat{U}_{\ell}\right) \leq \operatorname{cd}_{p}\left(\hat{G}_{\ell}\right) \leq 4$. Finally $\operatorname{dim}_{\mathbb{F}_{p}} H^{i}\left(\hat{U}_{\ell}, \mathbb{F}_{p}\right)=$ $\operatorname{dim}_{\mathbb{F}_{p}} H_{i}\left(\widehat{U}_{e}, \mathbb{F}_{p}\right)$ for all $i$ by Pontryagin duality. Thus

$$
\begin{aligned}
\sum_{0 \leq i \leq 4}(-1)^{i} \operatorname{dim}_{\mathbb{F}_{p}} H_{i}\left(\hat{U}_{\mathcal{C}}, \mathbb{F}_{p}\right) & =\sum_{0 \leq i \leq 4}(-1)^{i} \operatorname{dim}_{\mathbb{F}_{p}} H^{i}\left(\hat{U}_{e}, \mathbb{F}_{p}\right) \\
& =\sum_{0 \leq i \leq 4}(-1)^{i} \operatorname{dim}_{\mathbb{F}_{p}} H^{i}\left(U, \mathbb{F}_{p}\right)=\chi(U)=0
\end{aligned}
$$

and

$$
\begin{aligned}
2 \operatorname{dim}_{\mathbb{F}_{p}} H_{1}\left(\hat{U}_{\mathscr{C}}, \mathbb{F}_{p}\right)-\operatorname{dim}_{\mathbb{F}_{p}} H_{2}\left(\hat{U}_{\mathscr{C}}, \mathbb{F}_{p}\right) \\
\quad=2 \operatorname{dim}_{\mathbb{F}_{p}} H^{1}\left(\hat{U}_{\mathcal{C}}, \mathbb{F}_{p}\right)-\operatorname{dim}_{\mathbb{F}_{p}} H^{2}\left(\hat{U}_{\mathcal{C}}, \mathbb{F}_{p}\right) \\
=2 \operatorname{dim}_{\mathbb{F}_{p}} H^{1}\left(U, \mathbb{F}_{p}\right)-\operatorname{dim}_{\mathbb{F}_{p}} H^{2}\left(U, \mathbb{F}_{p}\right)=2 .
\end{aligned}
$$

4. Finally, if $G$ is $p$-good, then $\mu_{i, U}$ is the composition of the maps

$$
H^{i}\left(\hat{U}_{\mathcal{C}}, \mathbb{F}_{p}\right) \rightarrow H^{i}\left(\hat{G}_{\mathcal{C}}, \mathbb{F}_{p}[G / U]\right) \rightarrow H^{i}\left(G, \mathbb{F}_{p}[G / U]\right) \rightarrow H^{i}\left(U, \mathbb{F}_{p}\right),
$$

where $\mathcal{T}$ is the class of all finite $p$-groups, the first and the last map are Shapiro's isomorphisms and the middle one is an isomorphism since $G$ is $p$-good. Therefore, $\mu_{i, U}$ is an isomorphism.

\section{More on pro- $p$ completions}

Our first result is a more general version of Theorem 1 in the case of pro- $p$ completions. The new ingredient is the use of cohomology with coefficients in $\mathbb{F}_{p}\left[\left[\widehat{G}_{p}\right]\right]$ together with some results from [7] and [8].

Proof of Theorem 2. The conditions of Theorem 2 include the last two of the conditions of Theorem 1 but not the first one, i.e., we are not assuming that $2 \leq \operatorname{cd}\left(\widehat{G}_{p}\right)$. Note that the proof of Theorem 2 needed $2 \leq \operatorname{cd}\left(\widehat{G}_{p}\right)$ in order to show $H_{3}(\widehat{\mathcal{R}}) \not \npreceq \mathbb{F}_{p}$ (the only other possibility for $H_{3}(\widehat{\mathcal{R}})$ is 0 ), where $\widehat{\mathcal{R}}$ is the complex (2) for $m=4$ and $\widehat{G}_{p}$ is infinite (the last holds since $\widehat{G}_{p}$ is not virtually procyclic, hence is not virtually trivial). Then $H_{i}(\widehat{\mathcal{R}})=0$ for $i \neq 3$ and $H_{i}(\widehat{\mathcal{R}})$ is either 0 or $\mathbb{F}_{p}$.

Let $\mathcal{R}^{\text {op }}$ be a resolution as in (1) for $m=4$ but of the trivial left $\mathbb{Z}[G]$-module $\mathbb{Z}$ (recall that in (1) all modules are right $\mathbb{Z}[G]$ - modules). Then exchanging left with right modules we get similar results for the complex $\widehat{\widehat{R}^{\mathrm{op}}} \simeq \mathbb{F}_{p}\left[\left[\widehat{G}_{\ell}\right]\right] \otimes_{\mathbb{Z}[G]} \mathbb{Z}$, i.e., $H_{i}\left(\widehat{\mathcal{R}^{\mathrm{op}}}\right)=0$ for $i \neq 3$ and $H_{i}\left(\widehat{\mathcal{R}^{\mathrm{op}}}\right)=0$ is either 0 or $\mathbb{F}_{p}$. 
We claim that

$$
H_{3}\left(\widehat{\mathcal{R}^{\mathrm{op}}}\right) \simeq H^{1}\left(\widehat{G}_{p}, \mathbb{F}_{p}\left[\left[\widehat{G}_{p}\right]\right]\right) .
$$

Suppose that (13) holds and that $H_{3}\left(\widehat{\widehat{R}^{\mathrm{op}}}\right) \simeq \mathbb{F}_{p}$. Then $\operatorname{dim}_{\mathbb{F}_{p}} H^{1}\left(\widehat{G}_{p}, \mathbb{F}_{p}\left[\left[\widehat{G}_{p}\right]\right]\right)=1$ and by [7], Thm. $3, \widehat{G}_{p}$ is virtually $\mathbb{Z}_{p}$, a contradiction to condition a). Thus $\widehat{\widehat{R}^{o p}}$ is an exact complex and the proof of the dual version of Theorem 4 (exchanging left with right modules) completes the proof of Theorem 2.

Finally we prove (13). Let

$$
\mathcal{R}: 0 \rightarrow R_{4} \stackrel{\partial_{4}}{\rightarrow} R_{3} \stackrel{\partial_{3}}{\rightarrow} R_{2} \stackrel{\partial_{2}}{\rightarrow} R_{1} \stackrel{\partial_{1}}{\rightarrow} R_{0} \stackrel{\partial_{0}}{\rightarrow} \mathbb{Z} \rightarrow 0
$$

be the complex (1) for $m=4$.

Then $H^{i}(S)=H^{i}(G, \mathbb{Z}[G])$ is 0 for $i \neq 4$ and $\mathbb{Z}$ for $i=4$, where $S=$ $\operatorname{Hom}_{\mathbb{Z}[G]}\left(\mathcal{R}^{\text {del }}, \mathbb{Z}[G]\right)$ is the dual complex, i.e., $S$ is a complex of left $\mathbb{Z}[G]$-modules. Define $\mathcal{T}$ the complex obtained from $S$ by adding its unique non-trivial cohomology:

$$
\mathcal{T}: 0 \rightarrow S^{0} \rightarrow S^{1} \rightarrow S^{2} \rightarrow S^{3} \rightarrow S^{4} \rightarrow H^{4}(\mathcal{S})=\mathbb{Z} \rightarrow 0 .
$$

In particular the complex $\mathcal{T}$ is a projective resolution of the trivial left $\mathbb{Z}[G]$-module $\mathbb{Z}$. Consequently for

$$
\hat{\mathcal{T}}=\mathbb{F}_{p}\left[\left[\hat{G}_{p}\right]\right] \otimes_{\mathbb{Z}[G]} \mathcal{T}: 0 \rightarrow T^{0} \rightarrow T^{1} \rightarrow T^{2} \rightarrow T^{3} \rightarrow T^{4} \rightarrow \mathbb{F}_{p} \rightarrow 0
$$

we have

$$
H^{i}(\hat{\mathcal{T}})=\operatorname{Tor}_{4-i}^{\mathbb{Z}[G]}\left(\mathbb{F}_{p}\left[\left[\hat{G}_{p}\right]\right], \mathbb{Z}\right) \text { for } i \neq 4 \text { and } \quad H^{4}(\hat{\mathcal{T}})=0 .
$$

By the proof of Theorem 1,

$$
H_{i}(\widehat{\mathcal{R}})=0 \quad \text { for } i \neq 3,
$$

so $\hat{R}_{3} \rightarrow \hat{R}_{2} \rightarrow \hat{R}_{1} \rightarrow \hat{R}_{0} \rightarrow \mathbb{F}_{p} \rightarrow 0$ is exact, i.e., a partial projective resolution of the trivial $\mathbb{F}_{p}\left[\left[\widehat{G}_{p}\right]\right]$-module $\mathbb{F}_{p}$.

The deleted complex $\hat{\mathcal{T}}^{\text {del }}$ is the complex obtained from $\mathcal{T}$ by deleting the term $\mathbb{F}_{p}$. As in the proof of Theorem 4, we have

$$
\hat{\mathcal{T}}^{\text {del }} \simeq \operatorname{Hom}_{\mathbb{F}_{p}\left[\left[\widehat{G}_{p}\right]\right]}\left(\widehat{\mathcal{R}}^{\text {del }}, \mathbb{F}_{p}\left[\left[\widehat{G}_{p}\right]\right]\right) .
$$

Then by (16),

$$
\begin{aligned}
H^{1}\left(\widehat{G}_{p}, \mathbb{F}_{p}\left[\left[\widehat{G}_{p}\right]\right]\right) & =\operatorname{Ext}_{\mathbb{F}_{p}\left[\left[\widehat{G}_{p}\right]\right]}^{1}\left(\mathbb{F}_{p}, \mathbb{F}_{p}\left[\left[\widehat{G}_{p}\right]\right]\right) \\
& \simeq H^{1}\left(\operatorname{Hom}_{\mathbb{F}_{p}\left[\left[\widehat{G}_{p}\right]\right]}\left(\widehat{\mathcal{R}}^{\mathrm{del}}, \mathbb{F}_{p}\left[\left[\widehat{G}_{p}\right]\right]\right)\right) \\
& \simeq H^{1}\left(\hat{\mathcal{T}}^{\mathrm{del}}\right) \simeq \operatorname{Tor}_{3}^{\mathbb{Z}[G]}\left(\mathbb{F}_{p}\left[\left[\widehat{G}_{p}\right]\right], \mathbb{Z}\right) \simeq H_{3}\left(\widehat{\widehat{\mathcal{R}}^{\mathrm{op}}}\right),
\end{aligned}
$$

as required. 
Proof of Theorem 3. As in the proof of Theorem 1, we have

$$
H_{i}(\widehat{\mathcal{R}})=0 \quad \text { for } i=0,1,4,
$$

where $\widehat{\mathcal{R}} \simeq \mathcal{R} \otimes_{\mathbb{Z}[G]} \mathbb{F}_{p}\left[\left[\widehat{G}_{p}\right]\right], \mathcal{R}$ is the complex (1) for $m=4$ and again as in the proof of Theorem 1 for $U \in \mathscr{C}$ the map

$$
\varphi_{2, U}: H_{2}\left(U, \mathbb{F}_{p}\right) \rightarrow H_{2}\left(\widehat{U}_{p}, \mathbb{F}_{p}\right)
$$

is surjective.

Then by Lemma 3,

$$
\begin{aligned}
0 & \leq \operatorname{dim}_{\mathbb{F}_{p}} \operatorname{ker}\left(\varphi_{2, U}\right)=\operatorname{dim}_{\mathbb{F}_{p}} H_{2}\left(U, \mathbb{F}_{p}\right)-\operatorname{dim}_{\mathbb{F}_{p}} H_{2}\left(\widehat{U}_{p}, \mathbb{F}_{p}\right) \\
& \leq 2 \operatorname{dim}_{\mathbb{F}_{p}} H_{1}\left(U, \mathbb{F}_{p}\right)-2+m-2 \operatorname{dim}_{\mathbb{F}_{p}} H_{1}\left(\widehat{U}_{p}, \mathbb{F}_{p}\right) \\
& =m-2
\end{aligned}
$$

and hence

$$
\operatorname{dim}_{\mathbb{F}_{p}} \lim _{U \in \mathcal{C}} \operatorname{ker}\left(\varphi_{2, U}\right) \leq m-2 .
$$

Using the exact sequence

$0 \rightarrow \lim _{U \in \mathcal{C}} \operatorname{ker}\left(\varphi_{2, U}\right) \rightarrow \lim _{U \in \mathcal{C}} H_{2}\left(U, \mathbb{F}_{p}\right) \rightarrow\left(\lim _{U \in \mathcal{C}} H_{2}\left(\hat{U}_{p}, \mathbb{F}_{p}\right)\right)=0 \rightarrow \cdots$,

(3) and (19) we obtain that

$$
\begin{aligned}
\operatorname{dim}_{\mathbb{F}_{p}} H_{2}(\widehat{\mathcal{R}}) & =\operatorname{dim}_{\mathbb{F}_{p}} \operatorname{Tor}_{2}^{\mathbb{Z}[G]}\left(\mathbb{Z}, \mathbb{F}_{p}\left[\left[\widehat{G}_{e}\right]\right]\right) \\
& =\operatorname{dim}_{\mathbb{F}_{p}} H_{2}(\widehat{\mathcal{R}}) \\
& =\operatorname{dim}_{\mathbb{F}_{p}} \lim _{U \in \mathcal{C}} H_{2}\left(U, \mathbb{F}_{p}\right) \\
& =\operatorname{dim}_{\mathbb{F}_{p}} \lim _{U \in \mathcal{C}} \operatorname{ker}\left(\varphi_{2, U}\right) \leq m-2<\infty .
\end{aligned}
$$

By (18), $\sum_{0 \leq i \leq 4}(-1)^{i} \operatorname{dim}_{\mathbb{F}_{p}} H_{i}\left(U, \mathbb{F}_{p}\right)=0=\sum_{0 \leq i \leq 4}(-1)^{i} \operatorname{dim}_{\mathbb{F}_{p}} H_{i}\left(\widehat{U}_{p}, \mathbb{F}_{p}\right)$ and $H_{1}\left(U, \mathbb{F}_{p}\right) \simeq H_{1}\left(\widehat{U}_{p}, \mathbb{F}_{p}\right)$ we obtain that $\operatorname{dim}_{\mathbb{F}_{p}} H_{3}\left(U, \mathbb{F}_{p}\right)-\operatorname{dim}_{\mathbb{F}_{p}} H_{3}\left(\widehat{U}_{p}, \mathbb{F}_{p}\right)$ equals

$$
\begin{aligned}
& \sum_{0 \leq i \leq 4, i \neq 3}(-1)^{i}\left(\operatorname{dim}_{\mathbb{F}_{p}} H_{i}\left(U, \mathbb{F}_{p}\right)-\operatorname{dim}_{\mathbb{F}_{p}} H_{i}\left(\widehat{U}_{p}, \mathbb{F}_{p}\right)\right) \\
\quad= & \sum_{i=2,4}\left(\operatorname{dim}_{\mathbb{F}_{p}} H_{i}\left(U, \mathbb{F}_{p}\right)-\operatorname{dim}_{\mathbb{F}_{p}} H_{i}\left(\widehat{U}_{p}, \mathbb{F}_{p}\right)\right) \\
& \leq \operatorname{dim}_{\mathbb{F}_{p}} H_{4}\left(U, \mathbb{F}_{p}\right)-\operatorname{dim}_{\mathbb{F}_{p}} H_{4}\left(\widehat{U}_{p}, \mathbb{F}_{p}\right)+m-2 \\
& =m-1-\operatorname{dim}_{\mathbb{F}_{p}} H_{4}\left(\widehat{U}_{p}, \mathbb{F}_{p}\right) \\
& \leq m-1<\infty .
\end{aligned}
$$


Lemma 5. For $U \in \mathcal{C}$ and for the canonical map

$$
\varphi_{3, U}: H_{3}\left(U, \mathbb{F}_{p}\right) \rightarrow H_{3}\left(\widehat{U}_{p}, \mathbb{F}_{p}\right)
$$

we have

$$
\operatorname{dim}_{\mathbb{F}_{p}} \operatorname{coker}\left(\varphi_{3, U}\right)=\operatorname{dim}_{\mathbb{F}_{p}} H_{3}\left(\hat{U}_{p}, \mathbb{F}_{p}\right)-\operatorname{dim}_{\mathbb{F}_{p}} \operatorname{im}\left(\varphi_{3, U}\right) \leq \operatorname{dim}_{\mathbb{F}_{p}} H_{2}(\widehat{\mathcal{R}})
$$

Proof. In order to prove (22) consider a short exact sequence of complexes of $\mathbb{F}_{p}\left[\left[\widehat{U}_{p}\right]\right]-$ modules

$$
0 \rightarrow \widehat{\mathcal{R}} \rightarrow \mathcal{Q} \rightarrow \mathcal{S} \rightarrow 0,
$$

where all modules in $S$ positioned in dimension $\leq 2$ are $0, S$ is a shifted profinite deleted projective resolution of the $\mathbb{Z}_{p}\left[\left[\widehat{U}_{p}\right]\right]$-module $H_{2}(\widehat{\mathcal{R}})$, i.e., the first non-zero projective in $S$ is in dimension 3 and

$$
H_{i}(\mathcal{Q})=0 \quad \text { for } i \leq 2 .
$$

Furthermore there is a short exact sequence of profinite $\mathbb{F}_{p}\left[\left[\widehat{U}_{p}\right]\right]$ - complexes

$$
0 \rightarrow \mathcal{Q} \rightarrow \mathcal{V} \rightarrow \mathcal{W} \rightarrow 0
$$

where all modules in $W$ positioned in dimension $\leq 3$ are zero, $W$ is a shifted profinite deleted projective resolution of $H_{3}(\mathcal{Q})$, i.e., the first non-zero projective is in dimension 4 and

$$
H_{i}(\mathcal{V})=0 \text { for } i \leq 3 .
$$

Since $\widehat{\mathcal{R}} \hat{\bigotimes}_{\mathbb{F}_{p}\left[\left[\hat{U}_{p}\right]\right]} \mathbb{F}_{p}=\mathcal{R} \otimes_{\mathbb{Z}[G]} \mathbb{F}_{p}\left[\left[\widehat{G}_{p}\right]\right] \otimes_{\mathbb{F}_{p}\left[\left[\hat{U}_{p}\right]\right]} \mathbb{F}_{p} \simeq \mathcal{R} \otimes_{\mathbb{Z}[G]} \mathbb{F}_{p}[G / U]$ we have $H_{3}\left(\widehat{\mathcal{R}} \widehat{\otimes}_{\mathbb{F}_{p}\left[\left[\hat{U}_{p}\right]\right]} \mathbb{F}_{p}\right) \simeq H_{3}\left(G, \mathbb{F}_{p}[G / U]\right) \simeq H_{3}\left(U, \mathbb{F}_{p}\right)$, and since $\mathcal{V}^{(4)}$ is a partial profinite projective resolution of $\mathbb{F}_{p}$ over $\mathbb{F}_{p}\left[\left[\widehat{U}_{p}\right]\right]$ there is an isomorphism $H_{3}\left(\mathcal{V} \hat{\otimes}_{\mathbb{F}_{p}\left[\left[\hat{U}_{p}\right]\right]} \mathbb{F}_{p}\right) \simeq H_{3}\left(\hat{U}_{p}, \mathbb{F}_{p}\right)$. Under these isomorphisms the map $\varphi_{3, U}: H_{3}\left(U, \mathbb{F}_{p}\right) \rightarrow H_{3}\left(\hat{U}_{p}, \mathbb{F}_{p}\right)$ is the map

$$
f_{U}: H_{3}\left(\widehat{\mathcal{R}} \hat{\otimes}_{\mathbb{F}_{p}\left[\left[\hat{U}_{p}\right]\right]} \mathbb{F}_{p}\right) \rightarrow H_{3}\left(\mathcal{V} \widehat{\otimes}_{\mathbb{F}_{p}\left[\left[\hat{U}_{p}\right]\right]} \mathbb{F}_{p}\right),
$$

induced by the inclusion of $\widehat{\mathcal{R}}$ in $\mathcal{V}$.

Since the complexes $S$ and $W$ from (23) and (24) contain only projectives, we get exact sequences of complexes

and

$$
0 \rightarrow \widehat{\mathcal{R}} \hat{\otimes}_{\mathbb{F}_{p}\left[\left[\hat{U}_{p}\right]\right]} \mathbb{F}_{p} \rightarrow \mathcal{Q} \hat{\otimes}_{\mathbb{F}_{p}\left[\left[\hat{U}_{p}\right]\right]} \mathbb{F}_{p} \rightarrow S \widehat{\otimes}_{\mathbb{F}_{p}\left[\left[\hat{U}_{p}\right]\right]} \mathbb{F}_{p} \rightarrow 0
$$

$$
0 \rightarrow \mathcal{Q} \widehat{\otimes}_{\mathbb{F}_{p}\left[\left[\widehat{U}_{p}\right]\right]} \mathbb{F}_{p} \rightarrow \mathcal{V} \hat{\otimes}_{\mathbb{F}_{p}\left[\left[\widehat{U}_{p}\right]\right]} \mathbb{F}_{p} \rightarrow \mathcal{W} \widehat{\otimes}_{\mathbb{F}_{p}\left[\left[\widehat{U}_{p}\right]\right]} \mathbb{F}_{p} \rightarrow 0
$$


and the associated exact sequences in homology

$$
\begin{aligned}
\cdots & \rightarrow H_{3}\left(\widehat{\mathcal{R}} \hat{\bigotimes}_{\mathbb{F}_{p}\left[\left[\hat{U}_{p}\right]\right]} \mathbb{F}_{p}\right) \stackrel{f_{1, U}}{\longrightarrow} H_{3}\left(\mathcal{Q} \widehat{\otimes}_{\mathbb{F}_{p}\left[\left[\hat{U}_{p}\right]\right]} \mathbb{F}_{p}\right) \\
& \rightarrow H_{3}\left(\mathcal{S} \hat{\otimes}_{\mathbb{F}_{p}\left[\left[\hat{U}_{p}\right]\right]} \mathbb{F}_{p}\right)=\operatorname{Tor}_{0}^{\mathbb{F}_{p}\left[\left[\hat{U}_{p}\right]\right]}\left(H_{2}(\widehat{\mathcal{R}}), \mathbb{F}_{p}\right) \simeq H_{2}(\widehat{\mathcal{R}}) \bigotimes_{\mathbb{F}_{p}\left[\left[\hat{U}_{p}\right]\right]} \mathbb{F}_{p} \rightarrow \cdots
\end{aligned}
$$

and

$$
\begin{aligned}
\cdots \rightarrow H_{3}\left(\mathcal{Q} \hat{\otimes}_{\mathbb{F}_{p}\left[\left[\hat{U}_{p}\right]\right]} \mathbb{F}_{p}\right) \stackrel{f_{2, U}}{\longrightarrow} H_{3}\left(\mathcal{V} \hat{\otimes}_{\mathbb{F}_{p}\left[\left[\hat{U}_{p}\right]\right]} \mathbb{F}_{p}\right) \\
\longrightarrow H_{3}\left(\mathcal{W} \hat{\otimes}_{\mathbb{F}_{p}\left[\left[\hat{U}_{p}\right]\right]} \mathbb{F}_{p}\right)=0 \rightarrow \cdots
\end{aligned}
$$

Finally (22) follows from $f_{U}=f_{2, U} f_{1, U}, f_{2, U}$ is surjective and so

$$
\begin{aligned}
\operatorname{dim}_{\mathbb{F}_{p}} \operatorname{coker}\left(f_{U}\right) & \leq \operatorname{dim}_{\mathbb{F}_{p}} \operatorname{coker}\left(f_{1, U}\right) \\
& \leq \operatorname{dim}_{\mathbb{F}_{p}}\left(H_{2}(\widehat{\mathcal{R}}) \otimes_{\mathbb{F}_{p}\left[\left[\widehat{U}_{p}\right]\right]} \mathbb{F}_{p}\right) \\
& \leq \operatorname{dim}_{\mathbb{F}_{p}} H_{2}(\widehat{\mathcal{R}}) .
\end{aligned}
$$

Lemma 6. For all $i \geq 1$,

$$
\operatorname{Tor}_{i}^{\mathbb{Z}[G]}\left(\mathbb{Z}, \mathbb{F}_{p}\left[\left[\widehat{G}_{p}\right]\right]\right) \simeq H_{i}(\widehat{\mathcal{R}})=H_{i}\left(\mathcal{R} \otimes_{\mathbb{Z}[G]} \mathbb{F}_{p}\left[\left[\widehat{G}_{p}\right]\right]\right)
$$

is finite.

Proof. By (20), (21) and (22)

$$
\begin{aligned}
\operatorname{dim}_{\mathbb{F}_{p}} \operatorname{ker}\left(\varphi_{3, U}\right) & =\operatorname{dim}_{\mathbb{F}_{p}} H_{3}\left(U, \mathbb{F}_{p}\right)-\operatorname{dim}_{\mathbb{F}_{p}} \operatorname{im}\left(\varphi_{3, U}\right) \\
& \leq \operatorname{dim}_{\mathbb{F}_{p}} H_{3}\left(\widehat{U}_{p}, \mathbb{F}_{p}\right)+(m-1)-\operatorname{dim}_{\mathbb{F}_{p}} \operatorname{im}\left(\varphi_{3, U}\right) \\
& \leq \operatorname{dim}_{\mathbb{F}_{p}} H_{2}(\widehat{\mathcal{R}})+(m-1)<\infty .
\end{aligned}
$$

Then using the exact sequences

and

$$
0 \rightarrow \stackrel{\lim }{\longleftarrow}_{U \in \mathcal{C}} \operatorname{im}\left(\varphi_{3, U}\right) \rightarrow\left(\lim _{U \in \mathcal{C}} H_{3}\left(\widehat{U}_{p}, \mathbb{F}_{p}\right)\right)=0 \rightarrow \cdots
$$

$0 \rightarrow \lim _{U \in \mathcal{C}} \operatorname{ker}\left(\varphi_{3, U}\right) \rightarrow \lim _{\longleftarrow \in \mathcal{C}} H_{3}\left(U, \mathbb{F}_{p}\right) \rightarrow\left(\lim _{U \in \mathcal{C}} \operatorname{im}\left(\varphi_{3, U}\right)\right)=0 \rightarrow \cdots$, and by (26) we deduce that

$$
\begin{aligned}
& \operatorname{dim}_{\mathbb{F}_{p}} H_{3}(\widehat{\mathcal{R}})=\operatorname{dim}_{\mathbb{F}_{p}} \operatorname{Tor}_{3}^{\mathbb{Z}[G]}\left(\mathbb{Z}, \mathbb{F}_{p}\left[\left[\widehat{G}_{p}\right]\right]\right) \\
& =\operatorname{dim}_{\mathbb{F}_{p}} H_{3}(\widehat{\mathcal{R}}) \\
& =\operatorname{dim}_{\mathbb{F}_{p}} \lim _{U \in \mathcal{C}} H_{3}\left(U, \mathbb{F}_{p}\right) \\
& =\operatorname{dim}_{\mathbb{F}_{p}}{\underset{\lim }{\longleftarrow}}_{U \in \mathcal{C}} \operatorname{ker}\left(\varphi_{3, U}\right) \\
& \leq \operatorname{dim}_{\mathbb{F}_{p}} H_{2}(\widehat{\mathcal{R}})+(m-1)<\infty .
\end{aligned}
$$

Finally (17), (20) and (27) complete the proof. 
Consider the dual complex $\mathcal{M}=\operatorname{Hom}_{\mathbb{Z}[G]}\left(\mathcal{R}^{\text {del }}, \mathbb{Z}[G]\right)$. Define $\mathcal{T}$ the complex obtained from $\mathcal{M}$ by adding its unique non-trivial cohomology:

$$
\mathcal{T}: 0 \rightarrow M^{0} \rightarrow M^{1} \rightarrow M^{2} \rightarrow M^{3} \rightarrow M^{4} \rightarrow H^{4}(\mathcal{M})=\mathbb{Z} \rightarrow 0 .
$$

In particular the complex $\mathcal{T}$ is a projective resolution of the trivial left $\mathbb{Z}[G]$-module $\mathbb{Z}$ and as before we define $\hat{\mathcal{T}}=\mathbb{F}_{p}\left[\left[\widehat{G}_{p}\right]\right] \otimes_{\mathbb{Z}[G]} \mathcal{T}$. Then

$$
\begin{gathered}
\hat{\mathcal{T}}^{\mathrm{del}} \simeq \operatorname{Hom}_{\mathbb{F}_{p}\left[\left[\widehat{G}_{p}\right]\right]}\left(\widehat{\mathcal{R}}^{\mathrm{del}}, \mathbb{F}_{p}\left[\left[\widehat{G}_{p}\right]\right]\right), \\
H^{i}\left(\operatorname{Hom}_{\mathbb{F}_{p}\left[\left[\widehat{G}_{p}\right]\right]}\left(\widehat{\mathcal{R}}^{\mathrm{del}}, \mathbb{F}_{p}\left[\left[\widehat{G}_{p}\right]\right]\right)\right) \simeq H^{i}\left(\hat{\mathcal{T}}^{\mathrm{del}}\right) .
\end{gathered}
$$

As in the proof of Theorem 2 , let $\widehat{\widehat{R}^{\circ p}}$ be the version of $\widehat{\mathcal{R}}$ exchanging right with left modules. Then by the dual version of (25) (i.e., exchanging left with right modules)

$$
H^{i}\left(\hat{\mathcal{T}}^{\mathrm{del}}\right) \simeq \operatorname{Tor}_{4-i}^{\mathbb{Z}[G]}\left(\mathbb{F}_{p}\left[\left[\widehat{G}_{p}\right]\right], \mathbb{Z}\right) \simeq H_{4-i}\left({\widehat{\mathcal{R}^{\text {opdel }}}}\right)
$$

is finite for all $i \neq 4$ and

$$
H^{4}\left(\hat{\mathcal{T}}^{\mathrm{del}}\right)=0 .
$$

Since the complex $S$ in (23), considered for $U=G$, contains only projectives, we get a short exact sequence of complexes

$$
\begin{aligned}
\operatorname{Hom}_{\mathbb{F}_{p}\left[\left[\widehat{G}_{p}\right]\right]}\left(S, \mathbb{F}_{p}\left[\left[\widehat{G}_{p}\right]\right]\right) & \rightarrow \operatorname{Hom}_{\mathbb{F}_{p}\left[\left[\widehat{G}_{p}\right]\right]}\left(\mathcal{Q}, \mathbb{F}_{p}\left[\left[\widehat{G}_{p}\right]\right]\right) \\
& \rightarrow \operatorname{Hom}_{\mathbb{F}_{p}\left[\left[\widehat{G}_{p}\right]\right]}\left(\widehat{\mathcal{R}}, \mathbb{F}_{p}\left[\left[\widehat{G}_{p}\right]\right]\right)
\end{aligned}
$$

and the corresponding long exact sequence in cohomology

$$
\begin{aligned}
\cdots & \rightarrow H^{1}\left(\operatorname{Hom}_{\mathbb{F}_{p}\left[\left[\widehat{G}_{p}\right]\right]}\left(\mathcal{S}, \mathbb{F}_{p}\left[\left[\widehat{G}_{p}\right]\right]\right)\right)=0 \rightarrow H^{1}\left(\operatorname{Hom}_{\mathbb{F}_{p}\left[\left[\widehat{G}_{p}\right]\right]}\left(\mathcal{Q}, \mathbb{F}_{p}\left[\left[\widehat{G}_{p}\right]\right]\right)\right) \\
& \rightarrow H^{1}\left(\operatorname{Hom}_{\mathbb{F}_{p}\left[\left[\widehat{G}_{p}\right]\right]}\left(\widehat{\mathcal{R}}, \mathbb{F}_{p}\left[\left[\widehat{G}_{p}\right]\right]\right)\right) \rightarrow H^{2}\left(\operatorname{Hom}_{\mathbb{F}_{p}\left[\left[\widehat{G}_{p}\right]\right]}\left(\mathcal{S}, \mathbb{F}_{p}\left[\left[\widehat{G}_{p}\right]\right]\right)\right)=0 \\
& \rightarrow H^{2}\left(\operatorname{Hom}_{\mathbb{F}_{p}\left[\left[\widehat{G}_{p}\right]\right]}\left(\mathcal{Q}, \mathbb{F}_{p}\left[\left[\widehat{G}_{p}\right]\right]\right)\right) \rightarrow H^{2}\left(\operatorname{Hom}_{\mathbb{F}_{p}\left[\left[\widehat{G}_{p}\right]\right]}\left(\widehat{\mathcal{R}}, \mathbb{F}_{p}\left[\left[\widehat{G}_{p}\right]\right]\right)\right) \\
& \rightarrow H^{3}\left(\operatorname{Hom}_{\mathbb{F}_{p}\left[\left[\widehat{G}_{p}\right]\right]}\left(\mathcal{S}, \mathbb{F}_{p}\left[\left[\widehat{G}_{p}\right]\right]\right)\right) \simeq \operatorname{Ext}_{\mathbb{F}_{p}\left[\left[\widehat{G}_{p}\right]\right]}\left(H_{2}(\widehat{\mathcal{R}}), \mathbb{F}_{p}\left[\left[\widehat{G}_{p}\right]\right]\right) \rightarrow \cdots
\end{aligned}
$$

Note that $\operatorname{Ext}_{\mathbb{F}_{p}\left[\left[\widehat{G}_{p}\right]\right]}^{0}\left(\mathbb{F}_{p}, \mathbb{F}_{p}\left[\left[\widehat{G}_{p}\right]\right]\right) \simeq H^{0}\left(\widehat{G}_{p}, \mathbb{F}_{p}\left[\left[\widehat{G}_{p}\right]\right]\right)=0$ since $\widehat{G}_{p}$ is infinite (remember that $\widehat{G}_{p}$ is not virtually procyclic, hence is not virtually trivial), where $\mathbb{F}_{p}$ is the trivial $\mathbb{F}_{p}\left[\left[\widehat{G}_{p}\right]\right]$-module. Then since $H_{2}(\widehat{\mathcal{R}})$ is finite, it has a filtration of $\mathbb{F}_{p}\left[\left[\widehat{G}_{p}\right]\right]$-modules with simple quotients, and up to isomorphism there is a unique simple $\mathbb{F}_{p}\left[\left[\widehat{G}_{p}\right]\right]$-module that is the trivial $\mathbb{F}_{p}\left[\left[\widehat{G}_{p}\right]\right]$-module $\mathbb{F}_{p}$, we obtain that $\operatorname{Ext}_{\mathbb{F}_{p}\left[\left[\widehat{G}_{p}\right]\right]}^{0}\left(H_{2}(\widehat{\mathcal{R}}), \mathbb{F}_{p}\left[\left[\widehat{G}_{p}\right]\right]\right)=0$.

The inclusion map $\widehat{\mathcal{R}} \rightarrow \mathcal{Q}$ induces isomorphisms

$$
H^{i}\left(\operatorname{Hom}_{\mathbb{F}_{p}\left[\left[\widehat{G}_{p}\right]\right]}\left(\mathcal{Q}, \mathbb{F}_{p}\left[\left[\widehat{G}_{p}\right]\right]\right)\right) \rightarrow H^{i}\left(\operatorname{Hom}_{\mathbb{F}_{p}\left[\left[\widehat{G}_{p}\right]\right]}\left(\widehat{\mathcal{R}}, \mathbb{F}_{p}\left[\left[\widehat{G}_{p}\right]\right]\right)\right) \quad \text { for } i=1,2,
$$


and by (29), (30), (31) and the fact that the 3-skeleton $Q^{(3)}$ is a partial profinite projective resolution of $\mathbb{F}_{p}$ over $\mathbb{F}_{p}\left[\left[\widehat{G}_{p}\right]\right]$ it follows that

$$
\begin{aligned}
H^{i}\left(\widehat{G}_{p}, \mathbb{F}_{p}\left[\left[\widehat{G}_{p}\right]\right]\right) & \simeq H^{i}\left(\operatorname{Hom}_{\mathbb{F}_{p}\left[\left[\widehat{\sigma}_{p}\right]\right]}\left(\mathcal{Q}, \mathbb{F}_{p}\left[\left[\widehat{G}_{p}\right]\right]\right)\right) \\
& \simeq H^{i}\left(\operatorname{Hom}_{\mathbb{F}_{p}\left[\left[\widehat{G}_{p}\right]\right]}\left(\widehat{\mathcal{R}}, \mathbb{F}_{p}\left[\left[\widehat{G}_{p}\right]\right]\right)\right)
\end{aligned}
$$

is finite for $i=1,2$.

Furthermore by [7], Thm. 3, and (32) either $H^{1}\left(\widehat{G}_{p}, \mathbb{F}_{p}\left[\left[\widehat{G}_{p}\right]\right]\right)=0$ or $\widehat{G}_{p}$ is virtually $\mathbb{Z}_{p}$; the latter cannot hold by assumption. Thus $H^{1}\left(\widehat{G}_{p}, \mathbb{F}_{p}\left[\left[\widehat{G}_{p}\right]\right]\right)=0$, and since $\widehat{G}_{p}$ has type $\mathrm{FP}_{2}$ over $\mathbb{F}_{p}$ (remember $G$ is $\mathrm{FP}_{\infty}$ ) by [8], Thm. 1, Cor. 1, and (32) it follows that

$$
\text { either } H^{2}\left(\widehat{G}_{p}, \mathbb{F}_{p}\left[\left[\widehat{G}_{p}\right]\right]\right)=0 \quad \text { or } \quad \widehat{G}_{p} \text { is virtually a pro- } p \mathrm{PD}_{2} \text { group. }
$$

In the first case we obtain by (29), (32) and (30) that

$$
\begin{aligned}
H_{i}\left(\widehat{\mathcal{R}^{\mathrm{op}}}\right) & \simeq H^{4-i}(\widehat{\mathcal{T}}) \\
& \simeq H^{4-i}\left(\operatorname{Hom}_{\mathbb{F}_{p}\left[\left[\widehat{G}_{p}\right]\right]}\left(\widehat{\mathcal{R}}, \mathbb{F}_{p}\left[\left[\widehat{G}_{p}\right]\right]\right)\right) \\
& \simeq H^{4-i}\left(\widehat{G}_{p}, \mathbb{F}_{p}\left[\left[\widehat{G}_{p}\right]\right]\right)=0
\end{aligned}
$$

for $i=2,3$.

By the dual version of (17) obtained after exchanging left with right modules we have $H_{i}\left(\widehat{\mathcal{R}^{\mathrm{op}}}\right)=0$ for $i=0,1,4$. This combined with (34) implies that $\widehat{\widehat{R}^{\mathrm{op}}}$ is exact, i.e., $\operatorname{Tor}_{i}^{\mathbb{Z}[G]}\left(\mathbb{F}_{p}\left[\left[\widehat{G}_{p}\right]\right], \mathbb{Z}\right)=0$ for all $i \geq 1$. After exchanging left with right modules in the proof of Corollary 1 we get that condition c) of Corollary 1 can be substituted with $\operatorname{Tor}_{i}^{\mathbb{Z}[G]}\left(\mathbb{F}_{p}\left[\left[\widehat{G}_{p}\right]\right], \mathbb{Z}\right)=0$ for all $i \geq 1$. Thus $\widehat{G}_{p}$ is an orientable pro- $p \mathrm{PD}_{4}$ group, a contradiction, and by (33), $\widehat{G}_{p}$ is virtually a pro- $p \mathrm{PD}_{2}$ group.

Finally for some $V \in \mathcal{C}$ the pro- $p$ group $\widehat{V}_{p}$ is a pro- $p \mathrm{PD}_{2}$ group, hence a Demushkin group. For such a group, we have that $\operatorname{dim}_{\mathbb{F}_{p}} H_{2}\left(\widehat{V}_{p}, \mathbb{F}_{p}\right)=1$. Since $2 \operatorname{dim}_{\mathbb{F}_{p}} H_{1}\left(\widehat{V}_{p}, \mathbb{F}_{p}\right)-\operatorname{dim}_{\mathbb{F}_{p}} H_{2}\left(\widehat{V}_{p}, \mathbb{F}_{p}\right) \leq m$ there is an upper bound on $\operatorname{dim}_{\mathbb{F}_{p}} H_{1}\left(\widehat{V}_{p}, \mathbb{F}_{p}\right)$, i.e., $\widehat{V}_{p}$ is a finite rank Demushkin group. The classification of all infinite Demushkin groups can be found in [3], [4], [9] and [13] and this classification implies that $\widehat{V}_{p}$ has infinite abelianization. In particular there is a normal closed subgroup $N$ of $\widehat{V}_{p}$ such that $\widehat{V}_{p} / N \simeq \mathbb{Z}_{p}$. Because every subgroup of infinite index in a Demushkin group is a free pro- $p$ group, $N$ is a free pro- $p$ group and a pro- $p$ group of finite rank, so $N=\mathbb{Z}_{p}$. Thus $\widehat{V}_{p}$ is $\mathbb{Z}_{p}$-by- $\mathbb{Z}_{p}$.

\section{References}

[1] R. Bieri, Homological dimension of discrete groups. 2nd ed., Queen Mary College Mathematical Notes, Queen Mary College, London 1981. Zbl 0357.20027 MR 0715779 
[2] K. S. Brown, Cohomology of groups. Graduate Texts in Math. 87, Springer-Verlag, New York 1994. Zbl 0584.20036 MR 1324339

[3] S. P. Demuškin, The group of a maximal p-extension of a local field. Izv. Akad. Nauk SSSR Ser. Mat. 25 (1961), 329-346. Zbl 0100.03302 MR 0123565

[4] S. P. Demuškin, On 2-extensions of a local field. Sibirsk. Mat. Zh. 4 (1963), 951-955; English transl. Amer. Math. Soc. Transl. (2) 50 (1966), 178-182. Zbl 0131.27001 MR 0161854

[5] D. H. Kochloukova, Pro-C completions of orientable $P D^{3}$-pairs. Preprint, Campinas 2007.

[6] D. H. Kochloukova and P. A. Zalesskii, Profinite and pro- $p$ completions of Poincaré duality groups of dimension 3. Trans. Amer. Math. Soc. 360 (2008), 1927-1949. Zbl 1143.20016 MR 2366969

[7] A. A. Korenev, Pro- $p$ groups with a finite number of ends. Mat. Zametki 76 (2004), 531-538; English transl. Math. Notes 76 (2004), 490-496. Zbl 1080.20024 MR 2112069

[8] A. A. Korenev, Cohomology groups of pro- $p$-groups with coefficients in a group ring and the virtual Poincaré duality. Mat. Zametki 78 (2005), 853-863; English transl. Math. Notes 78 (2005), 791-800. Zbl 1129.20032 MR 2249035

[9] J. P. Labute, Classification of Demushkin groups. Canad. J. Math. 19 (1967), 106-132. Zbl 0153.04202 MR 0210788

[10] J. Neukirch, A. Schmidt, and K. Wingberg, Cohomology of number fields. Grundlehren Math. Wiss. 323, Springer-Verlag, Berlin 2000. Zbl 0948.11001 MR 1737196

[11] A. Reznikov, Three-manifolds class field theory (homology of coverings for a nonvirtually $b_{1}$-positive manifold). Selecta Math. (N.S.) 3 (1997), 361-399. Zbl 0892.57012 MR 1481134

[12] L. Ribes and P. Zalesskii, Profinite groups. Ergeb. Math. Grenzgeb. (3) 40, SpringerVerlag, Berlin 2000. Zbl 0949.20017 MR 1775104

[13] J.-P. Serre, Structure de certains pro- $p$-groupes (d'après Demuškin). Sém. Bourbaki 15 (1962/63), Exp. No. 252; Sém. Bourbaki, Vol. 8, Exp. No. 252, 145-155, Soc. Math. France, Paris 1995. Zbl 0121.04404 MR 1611538

[14] J.-P. Serre, Galois cohomology. Springer-Verlag, Berlin 1997. Zbl 0902.12004 MR 1466966

[15] P. Symonds and T. Weigel, Cohomology of $p$-adic analytic groups. In New horizons in pro-p groups, Progr. Math. 184, Birkhäuser, Boston 2000, 349-410. Zbl 0973.20043 MR 1765127

[16] T. Weigel, On profinite groups with finite abelianizations. Selecta Math. (N.S.) 13 (2007), 175-181. Zbl 2330590 MR 2330590

Received September 24, 2007; revised April 9, 2008

D. H. Kochloukova, Department of Mathematics, State University of Campinas

(UNICAMP), Cx. P. 6065, 13083-970 Campinas, SP, Brazil

E-mail: desi@ime.unicamp.br 